\title{
Comportement mécanique à très long terme des mines et cavernes dans le sel gemme : loi de Norton-Hoff ou loi de Lemaître?
}

M. KARIMI-JAFARI

Laboratoire de Mécanique des solides

École polytechnique

91128 Palaiseau Cedex Berest@

Ims.polytechnique.fr

\section{B. BROUARD}

Brouard Consulting 101, rue du Temple 75003 Paris

Contact@ Brouard-Consulting.com
Pour rendre compte de l'évolution observée pendant les essais de fluage réalisés au laboratoire sur des éprouvettes de sel gemme, deux modèles de comportement sont couramment utilisés : le modèle de Lemaître-Menzel-Schreiner (L-M-S) et le modèle de Norton-Hoff $(\mathrm{N}-\mathrm{H})$. Le premier suppose que la vitesse de déformation sous chargement constant décroît indéfiniment au cours du temps, alors que le second admet que cette vitesse tend vers une valeur constante. L'analyse des micromécanismes à l'origine des déformations penche en faveur de la loi de $\mathrm{N}-\mathrm{H}$; en revanche les essais de laboratoire ne permettent pas de trancher entre les deux hypothèses, car les incertitudes dues aux fluctuations de la température, de l'hygrométrie ou de la charge appliquée sont du même ordre de grandeur que les vitesses observées en fin d'essai. Les observations faites dans des ouvrages souterrains intéressent parfois des durées plus longues, mais leur interprétation est compliquée par la géométrie de l'ouvrage, la présence de phases transitoires mécaniques bien plus longues que celles observées au laboratoire et le rôle que prennent les phénomènes autres que mécaniques. On montre pourtant qu'à long terme les deux modèles prédisent pour une caverne souterraine abandonnée des comportements qualitativement différents, ce qui suggère de retenir, au cas par cas, le modèle qui donne les prévisions les plus pessimistes.

Mots-clés : comportement mécanique du sel gemme, loi de fluage de Lemaître-Menzel-Schreiner, loi de fluage de Norton-Hoff, loi de fluage de Munson-Dawson.

\section{Long term mechanical behaviour of salt mines and salt caverns : Norton-Hoff constitutive law versus Lemaitre constitutive law}

\footnotetext{
1 In order to account for the results of creep tests performed on salt samples, two mechanical constitutive laws are considered: the Lemaitre-Menzel-Schreiner (L-M-S) law and the NortonHoff (N-H) law. The former implies that the strain rate becomes constant some time after a constant load has been applied on the sample; the latter assumes that the strain rate gradually decreases and vanishes to zero after an infinite period of time. The analysis of the micro-physical processes in which deformation at sample scale originates supports the first assumption. However laboratory tests do not allow to discriminate these two assumptions, as uncertainties due to temperature, hygrometry or the applied load are of the same order of magnitude than the strain rates observed at the end of the test.
} 
Observations performed in underground mines or caverns can be significantly longer than laboratory tests; however they are difficult to interprete, as transient phases generally are much longer and various phenomena other than mechanical often play a significant role in the observed evolutions. However, in the long term, the two models lead to different prediction when long term behaviour of a cavern is considered which strongly suggests selection of the model whose predictions are more unfavourable.

Key words : mechanical behaviour of salt, Lemaitre-MenzelSchreiner mechanical constitutive law, Norton-Hoff mechanical constitutive law, Munson-Dawsun mechanical constitutive law.

\section{1}

\section{Introduction}

Dans les régions densément peuplées d'Europe, l'extraction à grande échelle du fer, du sel gemme, de la potasse, du charbon ou des hydrocarbures appartient largement au passé. Elle a laissé des vides souterrains dont la stabilité et l'étanchéité suscitent parfois des inquiétudes. De nouveaux projets sont apparus, dans lesquels le sous-sol est utilisé pour stocker ou enfouir des hydrocarbures, des déchets, radioactifs ou non, du dioxyde de carbone. Le public demande à juste titre que la sûreté de ces ouvrages, mines abandonnées ou stockages, soit assurée de façon certaine et, s'il y a lieu, pendant de très longues périodes de temps. Un exemple est constitué par l'abandon des mines sèches de sel (Bérest et al., 2004) ou des cavités réalisées par dissolution dans une formation salifère (Bérest, 2008). Ce dernier sujet a suscité des recherches nombreuses, notamment celles coordonnées par un organisme qui rassemble la profession à l'échelle mondiale, le SMRI (Ratigan, 2003).

Un aspect important de ces recherches est le comportement mécanique à long terme (plusieurs siècles) des ouvrages abandonnés. La prévision est fondée sur l'observation des ouvrages et surtout sur l'extrapolation des résultats d'essais de laboratoire conduits pendant des durées inévitablement limitées (quelques mois à quelques années).

On observe que, lorsqu'une charge constante est appliquée sur une éprouvette de sel pendant plusieurs mois, la vitesse de déformation décroît régulièrement, quoique de plus en plus lentement. Il y a une vingtaine d'années certains auteurs ont conclu que cette vitesse devait s'annuler après un temps fini. Cette conclusion conduisait, du point de vue du dimensionnement des mines, à des propositions extrêmement optimistes, et contredites par les faits ; elle a été abandonnée. Aujourd'hui la plupart des auteurs estiment que, sous charge constante, la vitesse de déformation tend vers une valeur constante non nulle (Langer, 1984 ; Munson et Dawson, 1984 ; Lux et Heuserman, 1983 ; Cristescu et Hunsche, 1998). En France plusieurs auteurs considèrent au contraire que cette vitesse doit continuer à décroître pour s'annuler après un temps infini (Vouille et al., 1984 ; voir aussi Aubertin et al., 1998). Aït Chalal (1996) a montré que ces points de vue, illustrés par les lois de Munson-Dawson, d'une part, et LemaîtreMenzel-Schreiner, d'autre part, permettaient, au prix d'un ajustement des paramètres des deux lois, des descriptions pratiquement indiscernables des résultats d'un essai de fluage multipaliers d'un mois et demi de durée. Durup et Xu (1996) arrivent à la même conclusion mais montrent que les deux descriptions conduisent à des résultats notablement différents lorsqu'on considère le comportement d'une caverne de stockage exploitée pendant 10000 jours. Le présent article a pour objet de poursuivre cette discussion.
2

\section{Essais de fluage sur des éprouvettes de sel}

L'essai de fluage uniaxial consiste à appliquer sur une éprouvette cylindrique pendant une durée assez longue une force constante axiale (compression) et à mesurer l'évolution au cours du temps de la déformation axiale (variation relative de la hauteur) et de la déformation diamétrale (variation relative du diamètre). On s'efforce parfois (Wawersik et Preece, 1984) d'appliquer une contrainte axiale constante (en corrigeant la force appliquée de la variation d'aire de la section) mais c'est une procédure plus compliquée et dont on peut discuter le bien-fondé (la déformation diamétrale n'est pas toujours la même sur toute la hauteur de l'éprouvette). Elle a aussi l'inconvénient de compliquer la comparaison entre les résultats obtenus par des laboratoires différents.

Surtout lorsque les propriétés du matériau provenant d'un même horizon géologique varient sensiblement d'une éprouvette à l'autre, on effectue souvent des essais multipaliers en appliquant successivement des charges différentes, laissées constantes pendant chaque palier, afin d'étudier sur une même éprouvette l'effet de l'intensité de la charge appliquée en évitant la dispersion qui affecte les essais conduits sur des éprouvettes distinctes.

On peut également effectuer des essais de fluage dits triaxiaux en appliquant, en plus de la charge axiale, une pression de fluide dite " de confinement " transmise à la surface latérale de l'éprouvette par une mem brane souple.

Dans le cas du sel gemme - sans doute la roche qui a fait l'objet du plus grand nombre d'expérimentations de laboratoire - (et aussi de la potasse) ces essais conduisent aux conclusions suivantes :

1) le comportement mécanique est en général isotrope (il ne dépend pas, ou faiblement, de l'orientation initiale au sein du massif de l'éprouvette prélevée). Ce résultat est toutefois inexact dans des gisements de sel en couche comportant de très nombreux lits de maté riaux argileux ou anhydritiques ;

2) lorsqu'une charge est appliquée instantanément puis laissée constante sur une éprouvette, la déformation évolue au cours du temps : elle comporte une déformation élastique instantanée suivie d'une déformation différée qui s'effectue à vitesse décroissante ;

3) hormis la déformation élastique initiale, la transformation s'effectue à volume constant à condition que l'état de contrainte appliqué reste à l'intérieur d'un domaine de l'espace des contraintes dit " domaine contractant ) (Spiers et al., 1988). Le domaine contractant est limité par le critère d'apparition de la dilatance. Ce domaine contient les états de contrainte isotropes. 

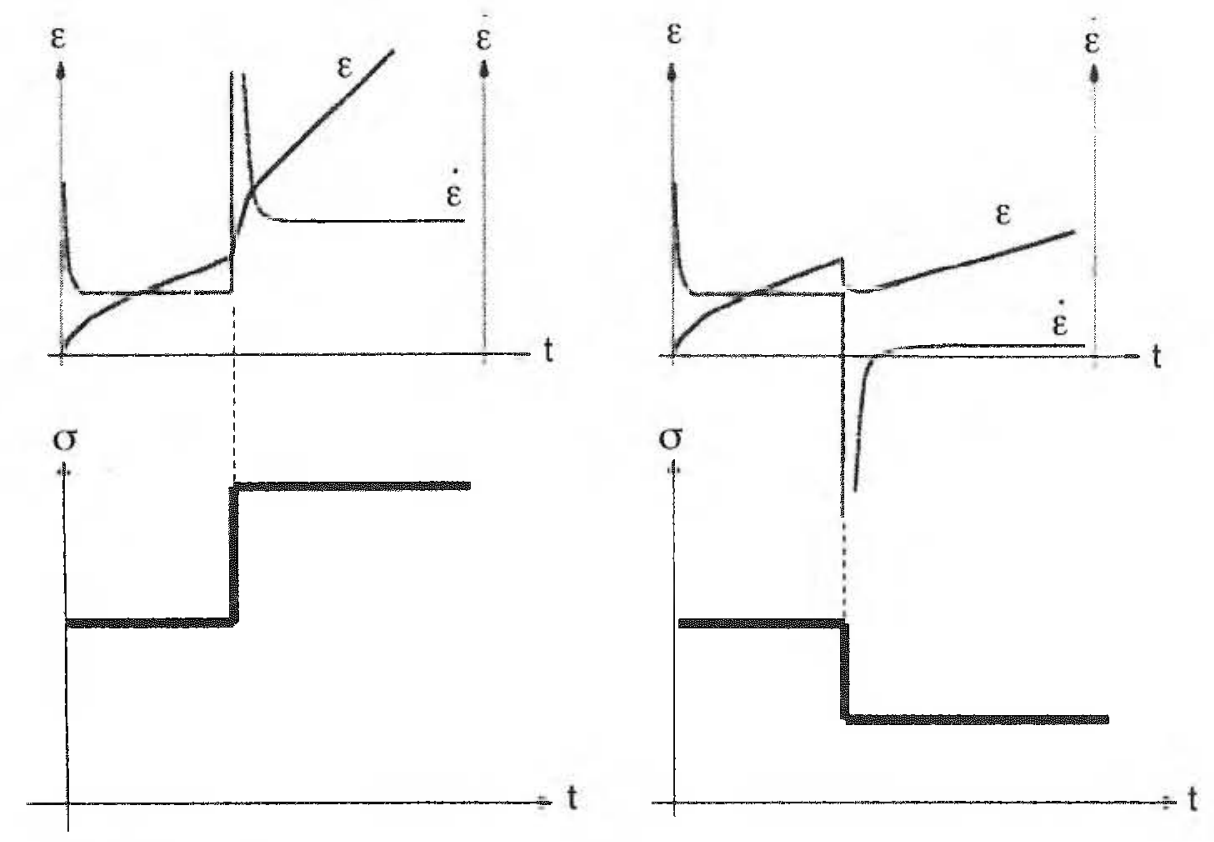

FIG. 1 Fluage transitoire pendant un essai uniaxial : cas de l'accroissement de charge (gauche) et de la réduction de charge (droite) ; $\varepsilon$ est la valeur absolue de la déformation axiale.

Transient creep during a uniaxial creep test : load increase (left) and load decrease (right) ; $\varepsilon$ is the absolute (positive) axial strain.

Une expression très simple pour l'étendue du domaine contractant a été proposée par Ratigan et al. (1991), $\sqrt{\mathrm{J}_{2}}<\mathrm{C} \mid \mathrm{I}_{1}$, où $J_{2}$ est le second invariant du tenseur déviateur des contraintes, $I_{1}$ est le premier invariant du tenseur des contraintes (en général $I_{1}<0$, les contraintes dans le massif rocheux étant des compressions) et $C$ est une constante pour un sel donné, $C=0,27$ pour les sels du Golfe du Mexique (des expressions plus précises, tenant compte de la distinction extension-compression et du cas particulier des charges uniaxiales, ont été proposées par DeVries et al. (2003). Quand le critère de dilatance est dépassé, la vitesse du son chute, la perméabilité et la porosité augmentent, la résistance diminue (Popp et al., 2007) ;

4) dans le domaine contractant, la déformation obtenue pour un chargement donné ne dépend que de l'écart entre la contrainte axiale appliquée et la contrainte de confinement ; autrement dit, pour le chargement considéré, elle ne dépend que du second invariant du tenseur déviateur des contraintes, qui coïncide dans ce cas avec cet écart ;

5) si on se place à des temps identiques (et suffisamment longs) après le début d'essais de fluage réalisés sous des charges mécaniques différentes, la vitesse de déformation varie comme l'écart entre la charge axiale appliquée et la contrainte de confinement élevé à une puissance de l'ordre de $n=3$ à 6 suivant le sel considéré (Brouard et Bérest, 1998) ;

6) la vitesse de déformation croît très vite avec la température de l'éprouvette

7) un changement de palier avec augmentation de la force appliquée engendre d'abord, hormis une (petite) déformation élastique instantanée, des vitesses de déformation rapides qui décroissent progressivement pour atteindre, après des temps comparables, des valeurs plus grandes que lorsque la force appliquée était plus faible ;
8) un changement de palier avec diminution de la force appliquée engendre d'abord, si la diminution est suffisamment importante ( $-30 \%$ typiquement), un accroissement de hauteur de l'éprouvette ( $($ fluage inverse $»$ ). Après un certain temps la vitesse de déformation change de signe pour atteindre des valeurs plus faibles, après des temps comparables, que lorsque la charge appliquée était plus grande.

Dans la décennie 1970-1980, certains auteurs ont cru pouvoir conclure que, non seulement la vitesse de déformation sous charge constante était décroissante, mais que de plus, au cours d'un essai de laboratoire comme dans un ouvrage souterrain, la déformation tendait à long terme vers une valeur finie. Le sel était donc, dans cette conception, un solide visqueux plutôt qu'un fluide. Cette thèse, parfois dite du strainhardening, est illustrée par un article de Serata (1968, p. 305), cité par Baar (1977) : « Any opening made in a salt media at a depth below $3000 \mathrm{ft}(900 \mathrm{~m})$ will suffer a large amount of plastic closure. However, regardless of the initial rate of closure, the creep rate also decreases exponentially to virtually zero as the opening reaches a stable condition ). Cette thèse a été critiquée par plusieurs auteurs (Langer, 1984), parfois avec une certaine virulence (Baar, 1977) : d'une part, elle est infirmée par les essais même très longs, d'autre part, elle n'est pas confirmée par les observations faites dans les mines où les piliers des quartiers exploités par chambres et piliers continuent à se déformer plusieurs décennies ou même plus d'un siècle après qu'ils ont été ouverts. De plus un tel point de vue conduisait à justifier un dimensionnement très optimiste des mines, « rock mechanics is not a magic wand which can be waved over a 50-ft pillar to turn it into a 150-ft pillar » (Abel, 1970, p. 207). Ce point de vue est abandonné aujourd'hui et tous les auteurs contemporains considèrent le sel comme un fluide visqueux (non newtonien et thermo-sensible) (Tijani et al., 1983). 


\section{Description des essais uniaxiaux et écriture d'une loi de comportement}

\section{1}

\section{Description des essais uniaxiaux}

On s'intéresse surtout par la suite à la description de la déformation observée au cours du temps lors d'un essai uniaxial d'un seul palier et de durée assez longue (plusieurs mois). La déformation thermo-élastique peut être décrite comme (les contraintes de compression sont négatives) :

$$
\begin{aligned}
& \dot{\varepsilon}_{11}^{\text {el }}+\dot{\varepsilon}_{11}^{\text {th }}=\dot{\sigma} / E+\alpha_{\text {th }} \dot{T} \\
& \dot{\varepsilon}_{22}^{\cdot d}+\dot{\varepsilon}_{22}^{\dot{t h}}=\dot{\varepsilon}_{33}^{\cdot e}+\dot{\varepsilon}_{33}^{\dot{b}}=-v \dot{\sigma} / E+\alpha_{\mathrm{th}} \dot{T}
\end{aligned}
$$

où o est la contrainte appliquée dans la direction $1, E, v$ sont le module d'élasticité et le coefficient de Poisson, $\alpha_{\text {th }}$ est le coefficient de dilatation thermique linéaire, $T$ est la température (absolue). Cette formulation doit être complétée par une description de la déformation viscoplastique. Deux types de description sont couramment utilisés :

$$
\begin{aligned}
& \text { Description de Lemaître-Menzel-Schreiner (L-M-S) } \\
& \dot{\varepsilon}=\dot{\varepsilon}^{\text {el }}+\dot{\varepsilon}^{\text {th }}+\dot{\varepsilon}^{\text {vp }} \\
& \varepsilon_{11}^{* p}=-A^{*}(T, \Phi)|\sigma|^{n^{*}} t^{\alpha^{*}} \\
& \dot{\varepsilon}_{31}^{\text {vp }}+\dot{\varepsilon}_{22}^{v_{p}}+\dot{\varepsilon}_{33}^{v_{p}}=0
\end{aligned}
$$

où $t$ est le temps et $\Phi$ est l'hygrométrie relative ambiante (quand l'éprouvette n'est pas isolée de l'atmosphère de la pièce). Pour exprimer la dépendance de $A^{*}$ avec la température, à $\Phi$ constant, on utilise souvent l'expression : $A^{*}=A_{0}^{*} \exp (-Q / R T)$.

\section{Description de Norton-Hoff $(\mathrm{N}-\mathrm{H})$}

$$
\begin{aligned}
& \dot{\varepsilon}=\dot{\varepsilon}^{\text {el }}+\dot{\varepsilon}^{\text {th }}+\dot{\varepsilon}^{v p}\left(+\dot{\varepsilon}_{\pi}^{v p}\right) \\
& \dot{\varepsilon}_{\mathrm{ss}, 11}^{v p}=-\mathrm{A}(\mathrm{T}, \Phi)|\sigma|^{\mathrm{n}} \\
& \dot{\varepsilon}_{\mathrm{ss}, 11}^{v p}+\varepsilon_{\mathrm{ss}, 22}^{v p}+\dot{\varepsilon}_{\mathrm{ss}, 33}^{v p}=0
\end{aligned}
$$

La description de L-M-S décrit la totalité du comportement viscoplastique au moyen de trois constantes seulement, $A^{*}$, $n^{*}$ et $\alpha *$ (pour une évolution isotherme). La description de N-H utilise deux constantes ( $A$ et $n$ ) mais elle ne décrit que le fluage établi et il est nécessaire de la compléter par une formulation de l'évolution transitoire initiale (ou $\dot{\varepsilon}_{\mathrm{tr}}^{\mathrm{vp}}$ ). La différence essentielle est que L-M-S prédit une diminution indéfinie - quoique progressivement très lente, car $\alpha^{*}<1-$ de la vitesse de déformation, alors que N-H prédit que la vitesse de déformation tend vers une valeur constante pour un essai dans lequel la contrainte applicuée (et les autres paramètres qui peuvent influencer la vitesse) restent constants. L'article est consacré à une comparaison de ces deux hypothèses.

\section{2.}

\section{Formulation d'une loi de comportement}

Les relations proposées plus haut décrivent un essai particulier mais ne constituent pas un modèle de comporte- ment. La description de L-M-S fait intervenir explicitement le temps : elle est donc relative à un choix de l'instant initial. Les deux descriptions concernent un essai dans lequel l'état de contrainte est uniforme dans l'éprouvette et invariable au cours du temps. Il faut étendre ces descriptions à des situations moins particulières. Pour cela, on dispose des observations faites pendant les essais et de quelques guides heuristiques. Le matériau étant supposé isotrope, la vitesse de déformation viscoplastique ne peut dépendre du tenseur des contraintes que par l'intermédiaire des trois invariants scalaires de celui-ci. On suppose aussi qu'il existe un potentiel viscoplastique : le tenseur des vitesses de déformation est proportionnel à une dérivée d'un potentiel par rapport à l'état de contrainte. Cette hypothèse présente l'avantage de fournir un certain nombre de résultats généraux sur les évolutions de structures réalisées dans ce matériau. Ce potentiel viscoplastique ne peut dépendre du premier invariant du tenseur des contraintes, ou $l_{t}$, puisque les déformations se font à volume constant. Il ne dépend donc que des invariants $J_{2}$ et $J_{3}$ du tenseur déviateur des contraintes. On suppose en général qu'il ne dépend que de l'invariant $J_{\mathrm{j}}$. C'est évidemment une hypothèse assez forte (A la suite d'un essai in situ effectué avec un grand soin sur un piler dans une chambre souterraine du WIPP, au Nouveau-Mexique, dont les résultats s'écartaient notablement des prévisions, cette hypothèse avait été réexaminée. Des essais de laboratoire sur tube, dans lesquels l'état de contrainte n'est pas homogène, et qui permettent donc de mieux discriminer les lois de comportement proposées pour rendre compte des essais, avaient permis de montrer qu'un potentiel construit à partir du critère de Tresca - plutôt que du critère de Von Mises, qui ne dépend que de l'invariant $J_{2}$ - donnait un meilleur accord avec les observations (Munson et Fossum, 1989). Toutefois, cette formulation, plus délicate à mettre en cuuvre dans les calculs numériques, n'est guère utilisée). Les formulations les plus souvent retenues sont le modèle de Lemaître-Menzel-Schreiner et le modèle de Norton-Hoff.

\section{Modèle de Lemaître-Menzel-Schreiner}

Le temps est éliminé entre la déformation et la vitesse de déformation en introduisant une variable interne notée $\zeta$

$$
\dot{\varepsilon}_{i j}^{v p}=\frac{\partial}{\partial t}\left(\zeta^{\alpha^{*}}\right) \frac{\partial}{\partial \sigma_{i j}}\left(\sqrt{3 J_{2}}\right) \quad \xi=\left(A^{* 1 / n^{*}} \sqrt{3 J_{2}}\right)^{n t / \alpha^{*}}
$$

\section{Modèle de Norton-Hoff}

$$
\dot{\varepsilon}_{\mathrm{ij}}^{\mathrm{ss}}=\mathrm{A}\left(\sqrt{3 \mathrm{~J}_{2}}\right)^{\mathrm{n}} \frac{\partial}{\partial \sigma_{\mathrm{ij}}}\left(\sqrt{3 \mathrm{~J}_{2}}\right)
$$

Cette loi est un cas particulier de la loi de Lemaitre quand $\alpha^{*}=1$.

\section{Modèle de Munson-Dawson}

Comme la loi de N-H ne décrit que la déformation viscoplastique stationnaire, elle doit être complétée par une formulation de la déformation viscoplastique transitoire. Un exemple est la loi de Munson-Dawson (1984):

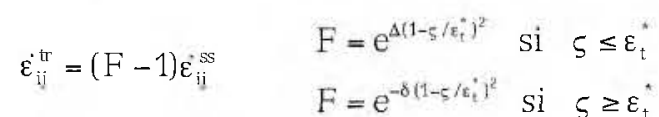




$$
\dot{\zeta}=(\mathrm{F}-1) \dot{\varepsilon}^{\mathrm{ss}}, \dot{\varepsilon}^{\mathrm{ss}}=\operatorname{Aexp}\left(-\frac{\mathrm{Q}}{\mathrm{RT}}\right)\left(\sqrt{3 \mathrm{~J}_{2}}\right)^{\mathrm{n}}
$$

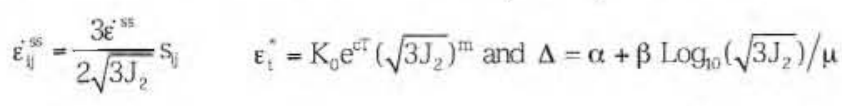

où $\mu$ est le module de cisaillement; cette loi signifie que, sous chargement constant, la déformation viscoplastique tend vers l'asymptote $\varepsilon^{\mathrm{vp}}=\varepsilon_{\mathrm{t}}^{*}+t \dot{\varepsilon}_{\mathrm{ss}}^{\mathrm{vp}}$.

\section{Identification des paramètres}

On peut considérer par exemple le cas du modèle de L-M-S. Gérard Vouille (communication personnelle) indique la procédure suivante d'identification des paramètres : on utilise la formulation (3), au début de l'essai le paramètre interne est nul; on peut, pour un jeu donné des paramètres de la loi de comportement et pour l'historique réel des contraintes appliquées et de la température, intégrer numériquement l'évolution $\dot{\zeta}=\dot{\zeta}(\mathrm{t})$ du paramètre interne, qui est continu même en cas de saut de la charge appliquée. La déformation totale pour sa part n'est pas continue mais il est facile de calculer le petit ( saut ) élastique de déformation engendré par un changement rapide de charge en fonction des paramètres élastiques $E$ et $v$. On obtient ainsi une réponse (calculée » de l'éprouvette pour une histoire donnée des charges appliquées et des températures, $\varepsilon_{\text {calc }}=\varepsilon_{\text {calc }}\left(t ; \alpha^{*}, n^{*}, A_{0}^{*}, E, v, Q / R\right)$, qui est fonction des paramètres de la loi de comportement. On compare alors cette réponse à la réponse mesurée " vraie ») soit $\varepsilon_{\text {mes }}=\varepsilon_{\text {mes }}$ ( $t$ ) et on cherche le meilleur choix de paramètres en minimisant la fonctionnelle

$$
\mathrm{F}\left(\alpha^{*}, \mathrm{n}^{*}, \mathrm{~A}_{0}^{*}, \mathrm{E}, v, \mathrm{Q} / \mathrm{R}\right)=\int_{0}^{T}\left(\varepsilon_{\text {calc }}-\varepsilon_{\text {res }}\right)^{2} \mathrm{dt}
$$

où $T$ est la durée totale de l'essai. L'algorithme de problème inverse donnant le jeu de valeurs des paramètres qui minimise la fonctionnelle a été mis au point par M. Tijani.

\section{Un commentaire sur les lois de comportement}

Un atout de la loi de L-M-S réside dans son économie remarquable de moyens : elle permet de décrire un essai de fluage isotherme de son début à sa fin au moyen de trois paramètres seulement (hormis les paramètres élastiques $E$ et $v$ ). Gérard Vouille (communication personnelle) fait d'ailleurs remarquer qu'elle rend compte de chargements complexes: lors d'un essai comportant l'application de paliers successifs avec charge croissante suivie de paliers successifs avec charge décroissante, il est d'observation constante que pour la même charge appliquée et après des temps comparables après le début du palier, la vitesse de déformation de l'éprouvette est plus lente à la « descente » (quand les charges décroissent) qu'à la « montée ») (quand les charges successives croissent). La loi de L-M-S rend bien compte de cet effet. La loi de N-H complétée par M-D aussi, mais c'est au prix d'un nombre plus élevé de paramètres.

La contrepartie de la simplicité est qu'on recherche un jeu de paramètres qui rende compte aussi bien du début de l'essai (pendant lequel les vitesses varient rapidement) que de la fin de celui-ci (quand les vitesses deviennent lentes). Or c'est surtout la fin de l'essai qui est susceptible de fournir une information utile du point de vue du comportement à long terme, sauf à supposer que la loi de L-M-S n'est pas un simple ajustement mais traduit des phénomènes mécaniques plus profonds, ce qu'aucune étude microphysique ne suggère à ce jour.

En revanche aucune des deux lois ne rend compte du fluage " inverse » (changement de signe transitoire de la vitesse de déformation à la suite d'une réduction substantielle de la contrainte appliquée) qui joue pourtant un rôle important pour l'interprétation des essais d'étanchéité des cavernes (Karimi-Jafari, 2007) marqués par un accroissement rapide et important de la pression de la caverne au début de l'essai et une augmentation transitoire de son volume, dont un exemple est donné au paragraphe 6.2.

\section{4 \\ Conséquence pour un ourrage du choix de la loi de comportement}

Un programme d'essais de laboratoire est nécessairement d'ampleur limitée. Il faut donc l'adapter aux conditions concrètes de contraintes, de température et d'hygrométrie attendues dans l'ouvrage. Toutefois, pour évaluer précisément les contraintes dans l'ouvrage, il faut déjà disposer d'une loi de comportement mécanique. Par ailleurs, le comportement mécanique d'une structure réalisée dans un matériau au comportement non linéaire peut présenter des caractères généraux notablement distincts de ceux présentés par une éprouvette au cours d'un essai triaxial, notamment parce que l'état de contrainte n'est ni homogène dans la structure ni constant au cours du temps.

La définition d'un programme d'essais de laboratoire est donc un processus itératif : les résultats des premiers essais et l'étude de la littérature suggèrent une première formulation de la loi de comportement qui permet de vérifier par un calcul d'ouvrage que les essais de laboratoire sont effectués dans la fenêtre de sollicitations (notamment de contraintes) auxquelles l'ouvrage souterrain sera effectivement soumis.

\section{1}

\section{Exemple d'une cavité sphérique}

On doit donc examiner le comportement mécanique d'une cavité souterraine pour les modèles de comportement envisagés. Pour un matériau élasto-viscoplastique dont le potentiel viscoplastique ne dépend que de l'état de contraintes, Mandel (1960) a montré, de manière en partie heuristique, que l'état de contrainte dans une structure soumise à un chargement mécanique constant tendait vers une distribution indépendante du temps. Pouya (1991) a généralisé ce résultat pour une famille de lois de comportement qui inclut les lois de L-M-S et N-H. Par exemple, le problème le plus simple posé par le comportement mécanique d'une cavité souterraine est celui dans lequel la pression intérieure, ou $\mathrm{P}_{c^{\prime}}$ plus faible que la pression géostatique, ou $\mathrm{P}_{x^{\prime}}$ est maintenue constante au cours du temps (cette pression est souvent, dans une mine, la pression nulle ou, dans une caverne, la pression dite halmostatique engendrée par une colonne de saumure saturée contenue dans le puits d'accès). On considère le cas idéalisé 
d'une caverne de forme sphérique, de rayon a, soumise à la pression intérieure $P_{c}$, et réalisée à une profondeur telle que l'état de contrainte naturel y est isotrope et égal à $\sigma_{i j}^{\infty}=-\mathrm{P}_{\infty} \delta_{i j}$. On a d'abord :

$$
\begin{aligned}
& \sigma_{\mathrm{rr}}>\sigma_{\theta \theta}=\sigma_{\varphi \varphi} \\
& \frac{\partial \sigma_{r T}}{\partial \mathrm{r}}+\frac{2}{r}\left(\sigma_{r \mathrm{rr}}-\sigma_{\theta \theta}\right)=0 \\
& \sigma_{r \mathrm{r}}(\infty)=-\mathrm{P}_{\infty} \quad \sigma_{\mathrm{rr}}(\mathrm{a})=-\mathrm{P}_{\infty}+\left(\mathrm{P}_{\infty}-\mathrm{P}_{\mathrm{c}}\right) \mathrm{H}(\mathrm{t})
\end{aligned}
$$

La première équation est une conséquence de la forme du chargement, et notamment de la condition $P_{c}<P_{\infty}$; la seconde relation est l'équation d'équilibre ; la troisième décrit les conditions à la limite. On doit de plus exprimer la loi de comportement élasto-viscoplastique. Du fait de la symétrie sphérique, les équations dans les deux directions orthoradiales sont identiques. Dans le cas de la loi de Norton-Hoff on a :

$$
\begin{aligned}
& \frac{\partial \dot{\mathrm{u}}}{\partial \mathrm{r}}=\frac{1}{\mathrm{E}}\left\{\dot{\sigma}_{\mathrm{rr}}-2 v \dot{\sigma}_{\theta \theta}\right\}+\mathrm{A}\left(\sigma_{\mathrm{rr}}-\sigma_{\theta \theta}\right)^{\mathrm{n}} \\
& \frac{\dot{\mathrm{u}}}{\mathrm{r}}=\frac{1}{\mathrm{E}}\left\{(1-v) \dot{\sigma}_{\theta \theta}-v \dot{\sigma}_{\mathrm{rr}}\right\}-\frac{\mathrm{A}}{2}\left(\sigma_{\mathrm{rr}}-\sigma_{\theta \theta}\right)^{\mathrm{n}}
\end{aligned}
$$

La solution stationnaire de ce problème pour les contraintes, atteinte après un temps très long sous chargement constant, peut s'écrire :

$$
\begin{aligned}
& \sigma_{\pi r}=-P_{\infty}+\left(P_{\infty}-P_{c}\right)\left(\frac{a}{r}\right)^{3 / n} \\
& \sigma_{\theta \theta}=-P_{\infty}+\left(1-\frac{3}{2 n}\right)\left(P_{\infty}-P_{c}\right)\left(\frac{a}{r}\right)^{3 / n} \\
& \sqrt{3 J_{2}}=3\left(P_{\infty}-P_{c}\right)\left(\frac{a}{r}\right)^{3 / n} / 2 n \\
& \dot{V} /\left.V\right|_{t=\infty}=-3 \frac{\dot{u}}{r}=-\frac{3}{2} A\left[\frac{3}{2 n}\left(P_{\infty}-P_{c}\right)\right]^{n}
\end{aligned}
$$

où $V$ est le volume de la caverne

\section{2}

\section{Conséquences pour un programme d'essais de laboratoire}

Il est intéressant de comparer la solution viscoplastique stationnaire (avec $n>1$ ) avec la solution élastique (obtenue en faisant $n=1$ ), car cette dernière donne la distribution initiale de contraintes dans le cas limite d'une création très rapide de la caverne (à l'instant initial, la pression dans la caverne passe instantanément de $\mathrm{P}=\mathrm{P}_{\infty}$ à $\mathrm{P}=\mathrm{P}_{c}<\mathrm{P}_{\mathrm{x}}$ ):

- les perturbations à l'état naturel de contrainte, induites par le creusement de la cavité, s'étendent beaucoup plus profondément au sein du massif dans le cas viscoplastique que dans le cas élastique (l'exposant du rayon est $-3 / n$ au lieu de -3 , et $n$ vaut typiquement de 3 à 6) ;

- pour une valeur fixée du rayon, le second invariant du tenseur des contraintes est divisé par un facteur $n$. Les contraintes déviatoriques sont donc nettement plus faibles que dans la solution élastique. Par exemple dans le cas d'une cavité creusée à $1000 \mathrm{~m}$ de profondeur et remplie de saumure, la pression géostatique est $P_{\infty}=22 \mathrm{MPa}$ et la pression halmostatique dans la caverne est $P_{c}=12 \mathrm{MPa}$. Dans un massif élastique, la contrainte déviatorique à la paroi serait $\sqrt{3 \mathrm{~J}_{2}(\mathrm{a})}=15 \mathrm{MPa}$; dans un massif de Norton-Hoff avec $n=3$, cette contrainte ne vaut plus, pour la solution stationnaire, que $\sqrt{3 \mathrm{~J}_{2}(\mathrm{a})}=5 \mathrm{MPa}$.
Le programme expérimental doit privilégier l'étude des effets de ces contraintes modérées. Les essais réalisés avec des valeurs plus élevées du déviateur ne sont toutefois pas inutiles, puisque de telles valeurs sont représenlatives de l'évolution transitoire de la Caverne, évolution qui peut être longue.

\section{3}

\section{Caractéristiques de l'épisode transitoire}

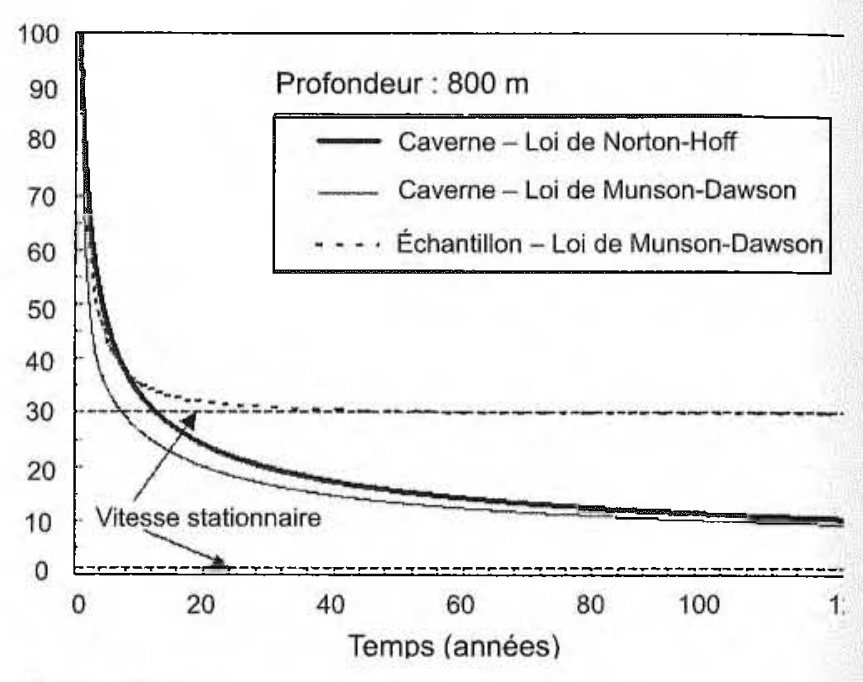

Fig. 2 Comparaison de la durée des transitoires pour une éprouvette de laboratoire et pour une caverne souterraine (d'après KarimiJafari, 2007).

Comparison of the transient period duration during a laboratory test and in an actual underground cavern lafter Karimi-Jafari, 20(17).

\section{Vitesse initiale de convergence}

Pour une caverne dont la pression intérieure est laissée constante au cours du temps, l'épisode transitoire compris entre la création de la caverne et le moment où la distribution stationnaire de contraintes est atteinte doit être discuté car il couvre pratiquement toute la durée de vie de I'ouvrage avant son abandon. On peut remarquer d'abord que, pour la loi de N-H, il existe un potentiel $\psi$ pour les déformations viscoplastiques :

$\dot{\varepsilon}_{\mathrm{ij}}^{\mathrm{si}}=\mathrm{A}\left(\sqrt{3 J_{2}}\right)^{\mathrm{n}} \frac{\partial}{\partial \sigma_{i j}}\left(\sqrt{3 J_{2}}\right)=\frac{\partial}{\partial \sigma_{i j}}\left|\mathrm{~A} \frac{1}{\mathrm{n}+1}\left(\sqrt{3 J_{2}}\right)^{n+1}\right| \equiv \frac{\partial}{\partial \sigma_{i j}} \psi\left(J_{2}\right)(9)$

On considère alors pour une évolution isotherme l'intégrale étendue à la totalité du massif :

$$
\int_{\Omega} \dot{\sigma}_{i j} \dot{\varepsilon}_{i j} \mathrm{~d} \Omega=\int_{\Omega} \dot{\sigma}_{i j} \dot{\varepsilon}_{i j}^{\mathrm{el}} \mathrm{d} \Omega+\int_{\Omega} \frac{\mathrm{d} \psi}{\mathrm{dt}} \mathrm{d} \Omega
$$

On montre facilement par une intégration par parties que l'intégrale de gauche est nulle, puisque les conditions en contrainte à la limite du domaine d'intégration sont invariables et que l'équation d'équilibre est évidemment vérifiée à tout instant. Or la première intégrale du membre de droite est manifestement positive ou nulle, de sorte que la seconde intégrale est négative ou nulle ; $\psi$ étant une fonction croissante du déviateur, celui-ci ne peut que décroître au moins en moyenne dans tout le massif. La démonstration s'étend au cas de L-M-S et de N-H complété par M-D. 
On peut être un peu plus précis dans le cas d'une caverne sphérique lorsqu'on choisit pour simplifier $v=1 / 2$ (incompressibilité élastique) car on a alors $\dot{u} / \mathrm{r}$ $=\mathrm{aa}^{2} / \mathrm{r}^{3}$ et la troisième relation du système (7) prend la forme

$$
\frac{\mathrm{a}^{2} \dot{\mathrm{a}}}{\mathrm{r}^{3}}=-\frac{1}{2 \mathrm{E}}\left(\dot{\sigma}_{\pi \mathrm{r}}-\dot{\sigma}_{\theta \theta}\right)-\frac{\mathrm{A}}{2}\left(\sigma_{r \mathrm{r}}-\sigma_{\theta \theta}\right)^{\mathrm{n}}
$$

Mais, à l'instant initial, le déviateur de contrainte suit la distribution élastique

$$
\sigma_{\pi T}-\left.\sigma_{\theta \theta}\right|_{t=0^{+}}=\frac{3}{2}\left(P_{\infty}-P_{c}\right) \frac{a^{3}}{r^{3}}
$$

De sorte qu'on peut, compte tenu de la condition d'équilibre, intégrer (11) à l'instant initial par rapport à $r$ entre l'infini et le rayon courant $r$. En particulier en choisissant $r=a$, où la contrainte radiale est égale au signe près à la pression intérieure invariable dans la caverne, on peut calculer la vitesse de convergence initiale :

$$
\dot{\mathrm{V}} /\left.\mathrm{V}\right|_{\mathrm{t}=0^{+}}=3 \dot{\mathrm{a}} /\left.\mathrm{a}\right|_{\mathrm{t}=0^{+}}=-\frac{3}{2 \mathrm{n}} \mathrm{A}\left[\frac{3}{2}\left(\mathrm{P}_{\infty}-\mathrm{P}_{\mathrm{c}}\right)\right]^{\mathrm{n}}\left(=\mathrm{n}^{\mathrm{n}-1} \dot{\mathrm{V}} /\left.\mathrm{V}\right|_{\mathrm{t}=\infty}\right)
$$

Celle-ci est beaucoup plus rapide que la vitesse de convergence associée à la solution stationnaire. Pendant l'épisode transitoire, cette vitesse diminue et la distribution de contrainte évolue de la distribution élastique initiale vers la distribution stationnaire.

Revenant alors au cas du point courant $r$, il est facile de voir que le déviateur des contraintes décroit à l'instant initial entre $r=a$ et $r=a n^{\frac{1}{3(n-1)}}$ et qu'il croît au-delà, comme le laisse d'ailleurs attendre la comparaison des déviateurs calculés pour l'état stationnaire et pour l'état élastique initial, respectivement. La démonstration s'étend facilement au cas de $\mathrm{N}-\mathrm{H}$ complété par $\mathrm{M}-\mathrm{D}$, au moins dans le cas particulier où $\beta=0$.

\section{Durée de l'épisode transitoire}

A la fois pour le comportement de $\mathrm{N}-\mathrm{H}$ et pour le comportement de L-M-S, Nguyen Minh et Pouya (1992) ont étudié l'évolution transitoire dans le cas d'une cavité sphérique soumise à une pression intérieure constante. Karimi-Jafari (2007) a examiné le cas de cavernes de forme quelconque. Ils ont établi que, pour les deux types de comportement, la solution ( stationnaire » ou " établie » n'est atteinte qu'après un temps très long. La figure 2 donne un exemple. On a représenté l'évolution de la vitesse de déformation axiale d'une éprouvette sur laquelle une charge de $\sigma=-8 \mathrm{MPa}$ était appliquée, pour la loi de N-H complétée par la modélisation du fluage transitoire de M-D. Pour la même loi de comportement on a représenté la vitesse de variation du volume relatif d'une caverne creusée à 800 mètres de profondeur et dans laquelle s'applique la pression halmostatique (en d'autres termes l'écart entre la pression géostatique et la pression dans la caverne est $\mathrm{P}_{\infty}-\mathrm{P}_{\mathrm{c}}=8 \mathrm{MPa}$ ). On a également représenté la solution du même problème quand la loi de N-H n'est pas complétée par la formulation de $\mathrm{M}-\mathrm{D}$ (aucun transitoire rhéologique n'est alors pris en compte). Les valeurs du module élastique et du coefficient de Poisson sont les mêmes pour tous les modèles, $E=25000 \mathrm{MPa}$ et $v=0,25$. Les paramètres du modèle de $\mathrm{N}-\mathrm{H}$ sont $\mathrm{A}=2 \times 10^{-11} / \mathrm{MPa}^{4} / \mathrm{jour}$ et $n=4$; les paramètres du modèle transitoire de $M-D$ sont $m=3, K_{0} e^{c r}=10^{-7} / \mathrm{MPa}^{3}, \delta=0,58$, et $\alpha=\Delta=10$.
On observe d'abord que l'épisode transitoire initial est beaucoup plus court pour l'éprouvette que pour la caverne ; il dure une quarantaine d'années pour l'éprouvette et plusieurs siècles pour la cavité. Dans le cas de la loi de N-H seule, il n'y aurait pas d'épisode transitoire pour une éprouvette alors qu'il en existe dans le cas de la caverne. Autrement dit, pour les temps courts, le choix de la loi rhéologique stationnaire de N-H conduit à un comportement non stationnaire d'une structure telle qu'une cavité souterraine. Ce phénomène est appelé « transitoire géométrique », parce qu'il tient à la lente redistribution des contraintes au sein du massif, par opposition au ( transitoire rhéologique », qui tient à l'introduction d'une composante transitoire dans l'écriture du modèle de comportement (Karimi-Jafari, 2007). On remarque aussi que, passé le premier siècle, il n'y a pas de différence très notable entre les évolutions de la caverne pour la loi de $\mathrm{N}-\mathrm{H}$ et pour la loi de $\mathrm{N}-\mathrm{H}$ complétée par M-D.

\section{4}

\section{Effets des grandes variations de volume}

On a vu qu'aux temps courts, l'évolution du volume de la caverne est transitoire, même si l'on retient le modèle de $\mathrm{N}-\mathrm{H}$. Considérons maintenant les temps très longs : si la cavité est un tant soit peu profonde, les déformations cumulées sont importantes et on ne peut se satisfaire de l'hypothèse des petites transformations. L'équation (8) doit alors être intégrée en tenant compte des modifications du volume de la cavité soit :

$$
\mathrm{V}(\mathrm{t}) / \mathrm{V}(0)=\exp \left(-\frac{3}{2} \mathrm{~A}\left[\frac{3}{2 \mathrm{n}}\left(\mathrm{P}_{\infty}-\mathrm{P}_{\mathrm{c}}\right)\right]^{\mathrm{n}} \mathrm{t}\right\}
$$

et, si la distribution de contrainte reste stationnaire, la vitesse de diminution du volume est une fonction décroissante du temps.

Le comportement mécanique d'une cavité souterraine présente donc certains caractères qualitatifs qui le distinguent du comportement d'une éprouvette de laboratoire; ce point sera repris auparagraphe 6.

\section{5}

\section{Apport des essais de laboratoire}

Les essais de fluage au laboratoire durent en général de trois mois à un an. Lorsqu'il s'agit d'essais multipaliers, la durée de chaque palier est de l'ordre de un à deux mois. Pour de telles durées, la vitesse de déformation est constamment décroissante. Ceci ne peut constituer en soi seul un argument décisif en faveur du modèle L-M-S. En effet le modèle de N-H doit être complété par une description du comportement transitoire rhéologique telle que celle de Munson-Dawson ; celle-ci prévoit aussi que l'épisode transitoire qui suit une modification de la contrainte appliquée a une durée au moins de l'ordre de celle de l'essai.

\section{1}

\section{Changement de forme de l'éprouvette}

L'éprouvette utilisée pour les essais est soigneusement rectifiée; sa forme initiale est cylindrique, et on peut attendre que l'état de contrainte soit homogène dans l'éprouvette, au moins à une échelle supérieure à celle des grains. Toutefois, lorsque les déforma- 
tions cumulées deviennent importantes, il est difficile d'assurer que le glissement au contact entre les faces inférieure et supérieure et les talons est parfait et les contraintes peuvent cesser d'être homogènes dans l'éprouvette. Cet effet a été discuté par Wawersick et Preece (1984). De même, Tijani (1987) a montré que dans un essai à vitesse de déformation contrôlée cet effet engendrait des contraintes de traction au voisinage du contact avec les talons. Une éprouvette au cours d'un essai réel de laboratoire est déjà une structure, siège d'effets complexes susceptibles de faire apparaître des non linéarités dans la relation déformation fonction du temps, même dans l'hypothèse de N-H.

\section{2}

\section{Effets de la température}

Pour discuter l'effet des fluctuations de température, on peut considérer l'ajustement des essais de Charpentier (1988) proposé par Pouya (1991) pour du sel de la formation supérieure d'Etrez (Ain) dans laquelle Gaz de France exploite des cavités de stockage de gaz. Pouya utilise un ajustement par une loi de Norton-Hoff, $\dot{\varepsilon}^{\cdot \mathrm{vp}}=-\mathrm{A}_{0} \exp \left(-\frac{\mathrm{Q}}{\mathrm{RT}}\right)|\sigma|^{\mathrm{n}}$ avec $A_{0}=0,64 \mathrm{MPa}^{-n} \mathrm{an}^{-1}$, $Q / R=4100 \mathrm{~K}, n=3,1$. Avec cette loi, un essai de fluage uniaxial pour une contrainte axiale appliquée de $\sigma=-10 \mathrm{MPa}$ doit conduire après deux à trois mois (durée des essais de Charpentier qui ont servi à l'ajustement) à une vitesse de déformation axiale de $\dot{\varepsilon}_{11}=-2.10^{-4} \mathrm{an}^{-1}$, soit environ $-0,610^{-11} \mathrm{~s}^{-1}$ ou $-5,510^{-7}$ jour $^{-1}$. Le coefficient de dilatation thermique du sel est de l'ordre de $\alpha_{t b}=4.10^{-5} /{ }^{\circ} \mathrm{C}$. Il n'est pas facile, même dans une pièce fermée, d'éviter des fluctuations journalières de température de l'ordre du degré Celsius. Autrement dit les fluctuations de la vitesse de déformation d'origine thermo-élastique sont de l'ordre de $\dot{\varepsilon}^{\text {th }}=\alpha_{\text {th }} \dot{\mathrm{T}} \approx 10^{-5}$ jour $^{-1}$, et sont beaucoup plus grandes que la vitesse moyenne de déformation en fin d'essai. On peut évidemment, lorsque l'essai est assez long, mesurer la température de la salle d'essai (ou mieux, de l'éprouvette) et rechercher une corrélation entre température et déformation pour quantifier les effets thermo-élastiques et corriger de ces effets la déformation observée. Ce n'est pas très facile. D'une part il existe un décalage temporel entre variation de température extérieure et déformation, la conduction thermique n'étant pas instantanée (pour une éprouvette de diamètre $D=10 \mathrm{~cm}$, le temps caractéristique pour l'uniformisation de la température dans l'éprouvette est de l'ordre de $\tau=D^{2} / \mathrm{k}$, où $\mathrm{k}=3.10^{-6} \mathrm{~m}^{2} \mathrm{~s}^{-1}$ est la diffusivité thermique du sel, soit $\tau=1 \mathrm{~h}$ ). D'autre part une variation de la température a également un effet sur la vitesse de déformation viscoplastique, $\delta \dot{\varepsilon}^{\mathrm{VP}} / \dot{\varepsilon}^{\mathrm{vp}}=\mathrm{Q} \delta \mathrm{T} / \mathrm{RT}^{2}$. Avec $\mathrm{Q} / \mathrm{R}=4100 \mathrm{~K}$, un accroissement de température de $\delta T=1{ }^{\circ} \mathrm{C}$ de la température moyenne, par exemple $T=286 \mathrm{~K}$, entraîne un accroissement relatif $\delta \dot{\varepsilon}^{\mathrm{vp}} / \dot{\varepsilon}^{\mathrm{vp}}=5 \%$ de la vitesse de fluage stationnaire. Enfin il est assez difficile, sauf conditions spéciales, de mesurer la température moyenne d'une éprouvette avec une résolution meilleure que $\delta T=1 / 10^{\circ} \mathrm{C}$, de sorte que l'incertitude pour le calcul de la vitesse sur une durée de $\delta t=30$ jours (par exemple) sera $2 \alpha \delta T / \delta t=2,710^{-7}$ jour $^{-1}$, c'est-à-dire la moitié de la vitesse qu'on souhaite mesurer : le risque est d'interpréter par de "l'écrouissage » une faible variation non détectée de la température moyenne.
D'une manière plus générale un changement de température de l'environnement de l'éprouvette peut affecter d'autres processus (par exemple l'hygrométrie relative) et influencer indirectement les phénomènes de fluage ; il n'est donc pas facile de distinguer tous les effets des variations de température, qui peuvent ne pas se réduire aux effets thermo-élastiques et à l'accélération de la vitesse de déformation viscoplastique, pris en compte pour leur part dans l'écriture du mudèle de comportement.

\section{3}

\section{Effet de l'hygrométrie}

L.es effets de l'hygrométrie sur la vitesse de fluage du sel sont également importants, comme l'ont souligné Horseman (1988) et Hunsche et Schulze (1996, 2002). Dans un essai uniaxial, l'éprouvette n'est pas toujours isolée de l'atmosphère de la pièce. Pour fixer les idées, sur le plateau de Saclay en région parisienne l'hygrométrie extérieure passe de $\Phi=70 \%-75 \% \mathrm{RH}$ l'été à $\Phi=80$ - $90 \%$ RH l'hiver. L'hygrométrie est sensiblement inférieure dans une galerie isolée d'une mine de sel, et probablement élevée dans une caverne de stockage de gaz. Hunsche et Schulze (20)2) ont proposé une relation entre vitesse de déformation stationnaire et humidité relative sous la forme:

$$
\dot{\varepsilon}_{\mathrm{s}}=\dot{\varepsilon}_{\mathrm{s} t}(1+\mathrm{W} \operatorname{sh}(\mathrm{q}(\mathrm{w}))
$$

où Ф est l'humidité relative (en \%RH); q et $w$ sont deux constantes respectivement égales à $q=0,1$ et $w=0,1$. Si on se place à une humidité relative moyenne de $\boldsymbol{w}_{0}=55 \%$ RH (cas de l'essai décrit à la figure 3), une variation d'humidité relative de $\delta \Phi=1 \%$ engendre une variation relative de la vilesse de déformation $\dot{\partial} \dot{\varepsilon} / \dot{\varepsilon}=0,09$ : il serait prématuré au vu des résultats bruts d'un essai de conclure à un écrouissage sans avoir soigneusement mesuré l'humidité relative. Dans un essai triaxial, l'éprouvelte est isolée de l'atmosphère extérieure et il est vraisemblable que la petite quantité d'air piégée entre l'éprouvette et la jaquette se mette à l'hygrométrie d'équilibre avec la saumure saturée contenue dans les pores de l'éprouvette, qui est de l'ordre de $75 \%$ RH et varie peu avec la température.

\section{4}

\section{Charge appliquée}

On peut faire une remarque andlogue pour la charge appliquée. Celle-ci résulte du fonctionnement d'un système hydraulique qui applique sur un piston une pression de fluide pilotée pour rester égale à une pression de consigne. Wawersik et Preece (1984) estiment, pour les essais (reconnus comme de très grande qualité) qu'ils ont effectués aux Sandia National Laboratories, que la contrainte déviatorique appliquée est contrôlée avec une précision de l'ordre de $\pm 2 \%$; cette valeur est relativement grande puisqu'elle doit être multipliée par l'exposant $n$ de la loi puissance pour obtenir l'incertitude induite sur la vitesse de déformation stationnaire. On doit de plus craindre que, sur une longue durée, ces systèmes connaissent une dérive du fait du vieillissement de l'électronique ou d'un grippage. 


\section{Un exemple}

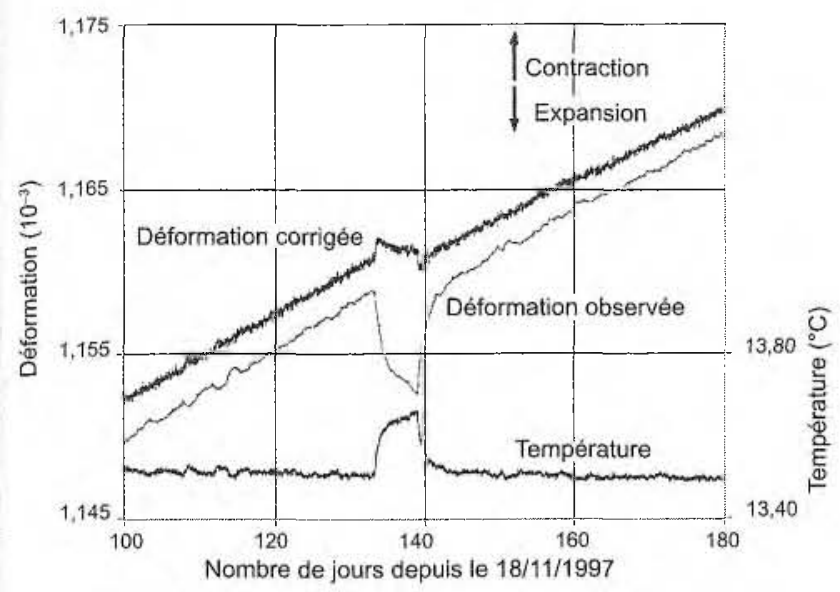

FIG. 3 Variations de la déformation et de la température observées et déformation corrigée des effets thermo-élastiques pendant l'essai $n^{\circ} 1$ dit de Varangéville. La charge appliquée était $0,108 \mathrm{MPa}$. Un décalage arbitraire de $2,510^{-6}$ de la déformation corrigée est appliqué pour faciliter la lecture (Bérest et al., 2005).

As-observed axial strain and temperature variations during the Varangéville Test $n^{\circ} 1$ together with the strain variations corrected for temperature variations. The applied load was $0.108 \mathrm{MPa}$. An arbitrary strain offset of $2.510^{-6}$ was applied to the corrected strain curve to make the figure easier to read (Bérest et al., 2005).

Un exemple est présenté sur la tigure 3. Le bâti de tluage à charge morte avait été placé. avec l'aimable concours de la société CSME, dans une gálerie souterraine profonde (160 $\mathrm{m}$ ) éloignée du système de ventilation de la mine pour bénéticier d'une température et d'une hygrométrie aussi constantes que possible (Bérest et al., 2(005). La figure représente 80 jours d'essai. Les tempéralures sont enregistrées toutes les deux minutes avec une résolution de l'ordre du millième de degré Celsius. Les fluctuations de tempéra. ture sunt de l'ordre de un à quelques centièmes de degré Celsius (ces fluctuations sont vraisemblablement dues aux variations de pression atmosphérique qui engendrent une compression ou une détente de l'air contenu dans la galerie (Perrier et al., 2001)I. De plus, au jour 132, une lampe est restée allumée par erreur, provoquant un accroissement de température de l'ordre de $0,15^{\circ} \mathrm{C}$ jusqu'à ce que la lampe brûle, six jours plus tard. On note la corrélation inverse très nette, due au comportement thermo-élastique, entre déformation et température. La correction thermo-élastique permet d'obtenir une courbe nettement plus régulière. On ne réussit pas toutefois à effacer complètement les elfets de l'épisode de la lampe allumée, sans doute à cause de l'influence de la température sur les déformations viscoplastiques, ou des variations de l'humidité relative. On observe pendant la période considérée (hiver) un lent refroidissement (de l'ordre de $0,\left(01^{\circ} \mathrm{C}\right.$ en 80 jours) qui rend la déformation observée (contraction) un peu plus grande que la déformation d'origine purement mécanique.

On retiendra en conclusion qu'il est important, pour la présentation des résultats d'un essai de fluage, de donner, en plus du tracé de la déformation au cours du temps, le tracé de l'évolution de la vitesse de déformation, de la température et de l'hygrométrie, et de préciser la date du début de l'essai.

\section{Apport des observations en place}

Pour trancher entre les formulations de N-H et de L-M-S, des essais très longs pourraient être utiles. Toutefois, il est difficile de mobiliser un bâti pendant une dizaine d'années, et le risque d'un incident qui ruine tous les efforts consentis est d'autant plus probable que l'essai est plus long. En revanche les ouvrages réels (caverne, mine, sondage) restent en général ouverts pendant plusieurs dizaines d'années et on peut, souvent à coût faible, y faire des observations pendant des durées longues. Celles-ci pourraient, en principe, permettre de discriminer les lois de comportement. En fait il n'en est rien.

La forme géométrique d'une chambre ou d'une caverne est moins simple que celle d'une éprouvette cylindrique, l'état de contraintes $n^{\prime} y$ est pas homogène, il n'atteint pas immédiatement une valeur constante (qui, au contraire, est réalisée instantanément dans un essai de fluage de laboratoire - au moins idéalement) et le massif contient en général de multiples hétérogénéités naturelles. On a établi ainsi au paragraphe 4.3 que, même lorsqu'on retient la loi de N-H qui ne décrit aucun fluage transitoire, une cavité dont la pression est portée à l'instant initial de la pression géostatique à une valeur constante plus basse est le siège d'un comportement global transitoire lié à la lente redistribution des contraintes dans le massif.

Plus généralement, un ouvrage est le siège de multiples phénomènes transitoires, dont les phénomènes transitoires mécaniques ne sont qu'un exemple ; la qualité des mesures, ou même la connaissance que l'on a des autres phénomènes, n'est pas toujours suffisante pour qu'on puisse extraire du signal enregistré la part qui revient à chacun d'entre eux.

\section{(1)}

\section{Essais à caverne fermée (Shut-in pressure test)}

Dans le cas des cavernes l'essai le plus simple consiste à fermer la caverne, remplie de saumure, et à suivre l'évolution naturelle de la pression au cours du temps. Même si cette évolution n'était régie que par des phénomènes mécaniques, l'interprétation serait délicate. En effet dans une caverne fermée le fluage du sel engendre une diminution de volume de la caverne, une contraction de la saumure contenue dans la caverne fermée et un accroissement de sa pression. La vitesse de fermeture décroit donc au cours du temps, quelle que soit la loi de comportement mécanique retenue, en raison de l'augmentation de la pression dans la caverne. L'évolution de la structure constituée par la caverne fermée étant transitoire par nature, il est difficile de distinguer nettement la part qu'y prend le comportement transitoire du matériau.

De plus, sauf peut-être pour une caverne laissée longtemps au repos, ou pour une caverne très profonde, l'évolution de la pression est en fait déterminée pour l'essentiel par le retour à l'équilibre thermique de la saumure contenue dans la caverne (les cavernes sont créées au moyen d'eau froide provenant de la surface ; la saumure qu'elles contiennent est donc initialement hors d'équilibre thermique avec le massif environnant; le réchauffement est très lent, il dure plusieurs décennies dans une caverne de volume supérieur 
à $100000 \mathrm{~m}^{3}$ ). Ce réchauffement engendre une dilatation de la saumure, et un accroissement de sa pression quand la caverne est fermée. Ce phénomène d'origine thermique est souvent prépondérant et masque le phénomène mécanique, dont la part est difficile à préciser. La figure 4 présente un exemple. Le 15 septembre 2005, la pression de la caverne fermée SPR2 du site de Carresse (exploitée par Total) est portée de 4,63 MPa à 4,83 MPa par injection d'huile dans l'espace annulaire (Brouard Consulting et al., 2006) (l'augmentation de la pression en tête de puits, représentée sur la figure 4, est bien entendu plus grande, la colonne d’huile formée étant plus légère que la colonne de saumure qu'elle remplace).

Pendant un mois environ la pression diminue, sous des effets déclenchés par l'accroissement initial de pression (les effets les plus importants sont la dissolution additionnelle : la concentration de la saumure à saturation est une fonction croissante de la pression et de la température et la dissolution s'effectue avec diminution de volume; et le fluage ( inverse » : le volume de la caverne augmente pendant quelques semaines, bien que la pression y soit plus faible que la pression géostatique. Une estimation de ces effets peut être trouvée dans Bérest et al., 2007). Puis la pression dans la caverne atteint un plateau avant de croître de nouveau (la courbe est tracée jusqu'en mars 2007) : cette croissance est due à l'échauffement de la saumure, qu'on a pu évaluer à $0,1^{\circ} \mathrm{C} /$ an pendant cette phase.

En l'absence d'échauffement, la pression chuterait car le volume de saumure perdu par perméation dans le massif est un peu plus grand que la perte de volume des cavernes par fluage (Brouard Consulting et al., 2006). Il est difficile dans ce contexte d'inférer une conclusion assurée quant à l'écriture de la loi de comportement mécanique pour les temps très longs bien que la perturbation thermique, source des incertitudes, soit très faible.
Lorsque les phénomènes thermiques sont devenus négligeables (c'est le cas après quelques années dans une petite caverne, par exemple de volume inférieur à $100(0) \mathrm{m}^{3}$, pour fixer les idées) les phénomènes de perméation (écoulement de la saumure vers le massif ou micro-fuites par la cimentation du puits) peuvent acquérir à leur tour une importance décisive et rendre difficile l'interprétation (Bérest et al., 2001).

\section{6}

\section{Essai à cavité ouverte (Liquid outflow test)}

On peut aussi envisager de réaliser un essai « à pression constante dans la caverne m en laissant la tête de puits ouverte et en recueillant la saumure fou l'hydrocarbure, dans certains cas) que le puits expulse naturellement. De manière équivalente, on peut laisser la tête de puits fermée et soutirer périodiquement de la cavité le volume de liquide nécessaire pour maintenir la pression dans la cavité égale à une valeur de consigne. De tels essais sont plus contraignants qu'un essai à cavité fermée, car il faut recueillir et mesurer au moins une tuis par jour le débit de saumure lou d'hydrocarburel expulsé ; ils ont l'avantage de présenter une certaine analogie avec un essai de fluage au laboratoire, pendant lequel la contrainte appliquée est maintenue constante, en évitant notamment certains phénomènes transitoires. De tels essais ont été décrits par ClercRenaud et Dubois (1980)), Hugout (1984), Gaulke et al. (2007). Lessai décrit par Hugout a été réalisé sur la petite $\left(8000 \mathrm{~m}^{3}\right.$ ) caverne EZ53 du site d'Etrez (Ain) exploité par Gaz de France (Fig. 5). Le puits contient un tube rempli de saumure saturée, ouvert en tête de puits ; l'annulaire, fermé en tête de puits, est rempli d'un hydrocarbure liquide, plus léger que la saumure, et dont la pression en tête de puits est donc supérieure à la pression atmosphérique. Trois mois après la fïn du

SPR2 - Évolution de la pression

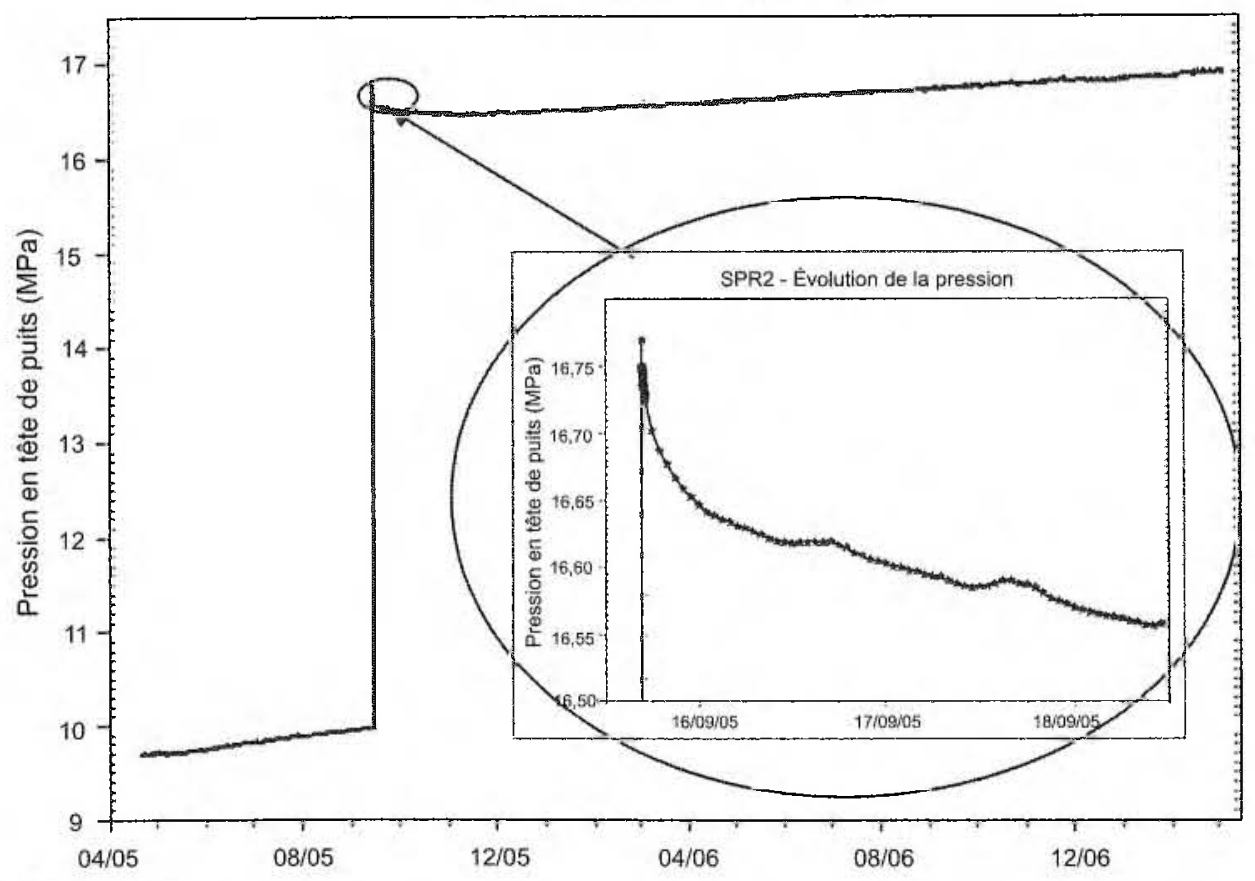

FIG. 4 Essai à caverne fermée sur la cavité SPR2 de Total à Carresse (PyrénéesAtlantiques).

Shut-in Pressure Test in the SPR2 cavern of the Carresse site (Pyrénées-Atlantiques) operated by Total. 
lessivage, au jour 93, le débit de saumure expulsé est de l'ordre de 100 litres/jour. On ouvre alors l'annulaire à la surface du sol: la pression dans la caverne chute de 11,4 MPa à 8,4 MPa, l'interface air/saumure descend dans le tube central, et de l'hydrocarbure est progressivement expulsée par l'annulaire (Fig. 6, cartouche de gauche). L'essai consiste à compter ce débit d'hydrocarbure. On observe un accroissement initial violent du débit expulsé, qui doit être attribué pour l'essentiel au fluage transitoire. Puis le débit décrôt, mais il n'est pas aisé de faire la part respective du transitoire mécanique rhéologique, du transitoire mécanique géométrique et du ralentissement du réchauffement. Ce dernier est certainement important car après le jour 254 on rétablit la pression initiale, un débit négatif est d'abord observé (dû au fluage ( inverse ) et à la dissolution complémentaire) puis le débit se stabilise à une valeur de l'ordre de 50 l/jour, soit sensiblement la moitié de la valeur observée avant l'essai : la mesure de la température dans la caverne montre que le réchauffement s'est considérablement ralenti (une analyse complète peut être trouvée dans Van Sambeek et al., 2005).

\section{3}

\section{Essai en sondage}

Un essai en sondage (ne débouchant pas dans une caverne) présente l'avantage d'éliminer pratiquement les effets thermiques: l'équilibre entre le massif et la saumure dans le sondage est très vite atteint compte tenu des faibles dimensions horizontales du trou (il s'agit d'un équilibre global, la saumure est le siège d'une convection naturelle due au gradient géothermique). Le même argument de dimension indique toutefois que les effets de perméation prennent un poids important: alors que la perte relative de volume par fluage est indépendante des dimensions, le débit relatif de perméation de saumure varie comme l'inverse du carré de celles-ci.

Dans un sondage fermé dont le découvert est creusé dans un milieu peu perméable et visqueux comme le sel, la pression converge en quelques années vers une pression constante, plus élevée que la pression halmostatique et dont la valeur résulte d'un équilibre entre la perméation, réglée par les propriétés hydrauliques, et la convergence, réglée par les propriétés mécaniques, de sorte qu'il ne renseigne qu'indirectement sur ces dernières.

Dans un sondage ouvert, comme dans une cavité ouverte, les effets de perméation sont moindres. A $1000 \mathrm{~m}$ de profondeur, on peut attendre une vitesse de déformation du découvert de l'ordre de $\dot{V} / V=-3.10^{-11} \mathrm{~s}^{-1}$, ou $5,510^{-7}$ jour $^{-1}$, soit un débit de saumure en tête de puits de l'ordre de $1,2 \mathrm{cl} / j$ our si le découvert a un volume de $4 \mathrm{~m}^{3}$. C'est un débit faible mais tout à fait mesurable, et l'essai mériterait d'être conduit. Le signal serait perturbé par les marées terrestres, dont la période est un peu supérieure à 12 heures, et qui engendrent des déformations de l'ordre de $\varepsilon=10^{-8}$ à $10^{-7}$.

\section{4}

\section{Mines}

Dans une mine exploitée par chambres et piliers on peut attendre, dans un quartier qui a cessé d'être exploité depuis plusieurs années, que la charge sur les piliers devienne constante. On peut ainsi bénéficier, en mesurant la hauteur des piliers et leur évolution, d'un essai ( naturel ) dans des conditions géométriques apparemment assez simples (et en bénéficiant d'une très grande constance de la température et de l'hygrométrie, si le quartier est éloigné de l'exploitation actuelle et du circuit de ventilation). Un ajustement de la loi de fluage des piliers d'une mine de potasse suivant la description de L-M-S a été proposé récemment par Campos de Orellana (1996). Dans une vieille mine comme celle de Varangéville, près de Nancy, exploitée par la compagnie CSME, on dispose de plus d'une estimation assez fiable de la hauteur initiale des piliers, creusés pour certains il y a plus d'un siècle. Mais ici aussi les conditions sont moins idéales que dans un laboratoire. Les piliers sont " encastrés » dans le toit et dans le mur, et ne réalisent donc pas les conditions de glissement parfait au contact entre l'éprouvette et les plateaux de la presse qu'on s'efforce de réaliser au laboratoire. De ce fait les piliers se déforment ( en tonneau ), et la distribution de contraintes est complexe au sein du pilier, Van Sambeek (1996), Laouafa et Ghoreychi (2006).
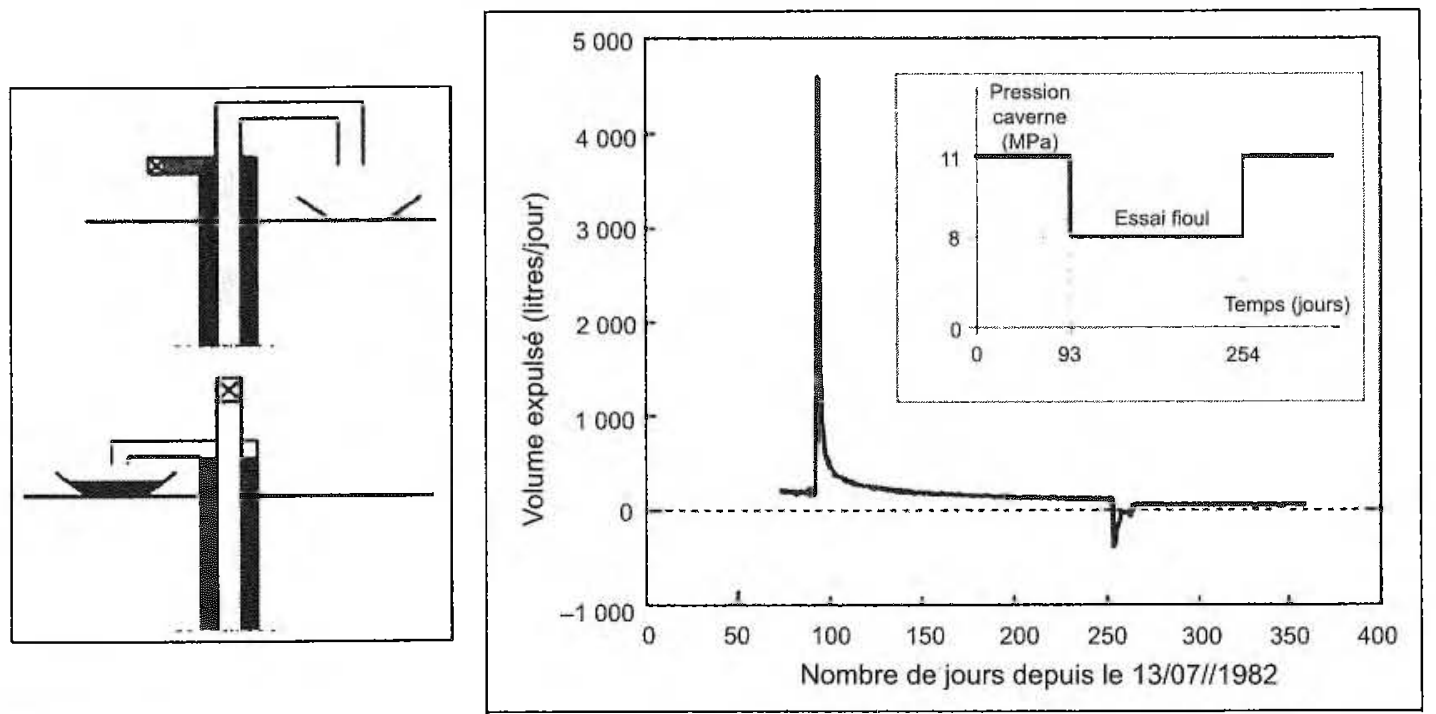

FG.5 Essai à cavité ouverte dans la caverne EZ53 de Gaz de France, d'après Hugout, 1984. Liquid outflow test in the Gaz de France EZ 53 cavern, after Hugout, 1984. 


\section{Apport des observations géologiques}

Les observations géologiques peuvent apporter des indications, d'autant que les formations salifères sont souvent affectées de déformations importantes. Notamment des dômes de sel se forment fréquemment à partir de couches qui étaient horizontales au moment du dépôt, sous l'effet des seules forces gravitaires : la compaction progressive des terrains sus-jacents rend leur poids volumique sensiblement plus grand que celui du sel, engendrant ainsi une situation instable : une intumescence quelconque au toit de la formation salifère tend à croître spontanément, et le sel remonte vers la surface du sol avec des vitesses qui peuvent être de l'ordre du mm/an ou même du cm/an. Les déplacements verticaux cumulés peuvent être d'ordre kilométrique. Il est toutefois difficile de tirer des conclusions définitives quant à la loi de comportement mécanique du sel, pour la même raison que dans le cas d'un ouvrage souterrain : on est très loin du cas élémentaire d'une éprouvette de laboratoire soumise à un chargement constant. Lorsque le dôme commence son ascension, les déséquilibres dus aux forces gravitaires, qui sont de l'ordre de grandeur de l'écart entre les poids volumiques multipliés par la hauteur du dôme, croissent avec le temps ; le dôme rencontre des obstacles divers (couches raides par exemple) qui peuvent ralentir pour un temps son développement. A supposer qu'on puisse reconstituer exactement la chronologie de la formation du dôme, il resterait des incertitudes quant à la part que prennent dans les étapes de cette chronologie la rhéologie du sel, l'accroissement des dimensions du dôme, la poursuite de la compaction, l'histoire des distributions de température et les circonstances extérieures comme l'érosion à la surface du sol.

\section{8}

\section{Micromécanismes}

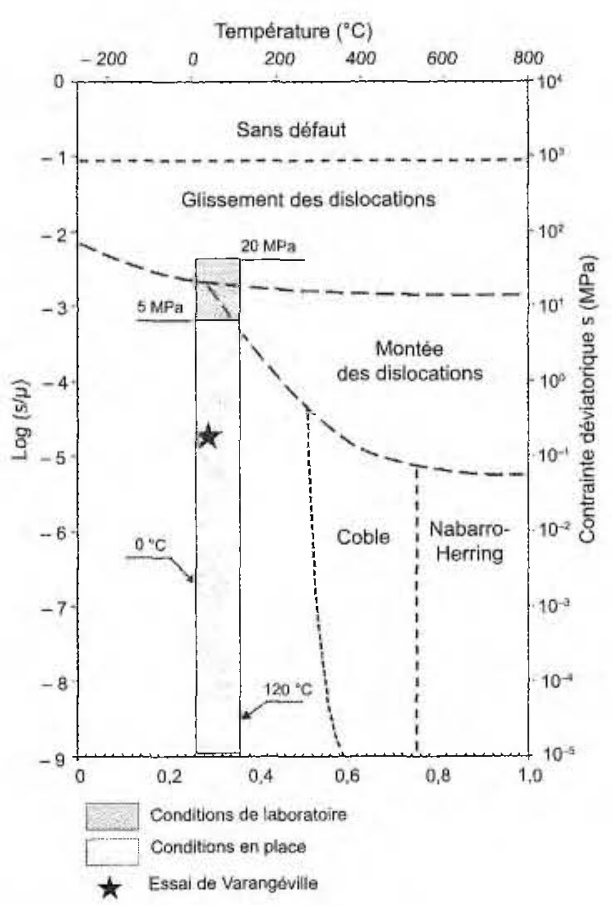

FG. 6 Carte de mécanismes, d'après Munson et Dawson, 1984.

Mechanism Map, after Munson and Dawson, 1984.
En principe, les prédictions relatives au comportement à long terme des roches devraient être basées sur une compréhension fine des mécanismes qui gouvernent ce comportement à petite échelle. Comme l'indique Langer (1984) : ( Reliable extrapolation of the creep equations over long period of time and at low deformation rates can only be carried out on the basis of deformation mechanisms. The construction of a deformationmechanism map is an essential preliminary. "De telles cartes des mécanismes de déformation sont présentées par Munson et Dawson (1984), Langer (1984), Blum et Fleischmann (1988). La température (absolue) est rapportée à la température de fusion $\left(T_{i}=1077 \mathrm{~K}\right.$ pour $\mathrm{NaCl}$ l et la contrainte déviatorique est rapportée au module de cisaillement $(\mu)$. Le sel cristallise en système cubique et un échantillon est constitué d'un très grand nombre de grains : les mécanismes sont a priori associés aux mouvements des dislocations, aux glissements suivant les directions données par le réseau cristallin et aux phénomènes affectant les joints de grains. Divers domaines apparaissent sur la carte, à l'intérieur desquels tel ou tel mécanisme de déformation est dominant (les frontières tracées correspondent au lieu des points où deux mécanismes ont une contribution égale à la vitesse de déformation). Les conditions de température et de contrainte dans un stockage de déchets radioactifs ou une caverne de stockage d'hydrocarbures sont inscrites dans le rectangle de la figure 6 , limité par $25^{\circ} \mathrm{C}$ et $200^{\circ} \mathrm{C}$, d'une part, 0,2 $\mathrm{MPa}$ et $20 \mathrm{MPa}$, d'autre part. Dans ce rectangle le mécanisme de déformation est "indétini », même si on dispose d'un grand nombre de données empiriques. Les auteurs cités attribuent un modèle de comportement de Norton-Hoff à ce domaine; Munson et Dawson indiquent qu'il n'y a pas de raison de penser que les mécanismes qui régissent le comportement transitoire soient distincts, de sorte que le fluage établi résulte d'un équilibre entre processus pour lequel aucun argument ne milite en faveur d'une modification au cours du temps. Malgré leur pertinence, ces arguments ne convaincront peutêtre pas complètement les ingénieurs, d'autant que d'autres mécanismes ont été proposés par Pennock et al. (2006) pour décrire le comportement sous très faible contrainte déviatorique (de l'ordre de 0.1 MPa pour fixer les idées).

\section{Comportement à très long terme d'une caverne fermée}

Hormis les deux paramètres élastiques $E$ el v, qui sont identiques quel que soit le modèle retenu, à température fixée le modèle de L.-M-S comporte trois constantes $\left(A^{*}, n^{*}, \alpha^{*}\right)$ et le modèle de N-H deux constantes ( $A$ et $n$ ) auxquelles il faut ajouter les constantes du modèle transitoire de $\mathrm{M}$-D. Dans un essai uniaxial sans diminution du chargement, quatre constantes de ce modèle jouent un rôle : $K_{w}, \mathrm{~m}$, $\alpha$ el $\beta$. Il est facile d'ajuster les trois constantes du modèle de L.-M-S et les six constantes du modèle de $\mathrm{N}$-H complété par M-D pour que les deux modèles donnent des descriptions pratiquement indiscernables du même essai de fluage. Un exemple est donné à la figure 7 : on a simulé un essai d'une durée de 100 jours, la température est de $45^{\circ} \mathrm{C}$ et la charge appliquée est $10 \mathrm{MPa}$. Le module élastique est $\mathrm{E}=25000 \mathrm{MPa}$, le coefficient de Poisson est $v=0,25$. Les paramètres du modèle de $\mathrm{L}$--M-S sont 
$\mathrm{A}^{*}=\mathrm{K}^{-\mathrm{n}^{*}} 5,6 \times 10^{-8} / \mathrm{MPa}^{4,82} / \mathrm{jour}^{0,058}, \mathrm{n}^{*}=4,82 \mathrm{et}$ $\alpha^{*}=0,058$. Pour le modèle de $\mathrm{N}-\mathrm{H}$ on a retenu $A=2,6 \times 10^{5} / \mathrm{MPa}^{5,5} /$ jour, $n=5,5$. Pour le modèle de M-D on a $K_{0}=3,3 \cdot 10^{-7}, m=3$, et $\beta=-7,74$.

On a ensuite considéré le problème d'une cavité fermée et abandonnée. Du point de vue de la protection de l'environnement, il est important de prévoir comment la pression de la saumure contenue dans la cavité évoluera au cours du temps. Il ne faut pas, notamment, qu'elle dépasse ou même approche de trop près la valeur de la pression géostatique, afin que soit écarté le risque d'une fracturation du massif de sel qui pourrait conduire à expulser de la saumure saturée vers des horizons aquifères.

Il a été montré qu'à très long terme (plusieurs siècles après la fermeture de la caverne) l'évolution de cette pression résulte de deux phénomènes aux effets antagonistes : la fermeture de la caverne, qui résulte du fluage du sel, d'autant plus lente que la pression dans la caverne est élevée; et la (micro) perméation de la saumure vers le massif de sel, qui est d'autant plus rapide que la pression est élevée. Lorsque ces deux phénomènes sont exactement équilibrés, la pression reste stationnaire (Bérest et al., 2001).

On admet en général que le débit de (micro) perméation est proportionnel à l'écart entre la pression de la caverne et la pression de pore dans le massif, ou, autrement dit, qu'il suit la loi phénoménologique de Darcy, même si les mécanismes de cette perméation sont incomplètement compris. Durup (1994) a vérifié, au cours d'un long essai dans un puits creusé dans la formation salifère d'Etrez, que cette hypothèse était raisonnable. Dans les calculs décrits plus loin on a retenu une valeur de la perméabilité égale à $\mathrm{k}_{\mathrm{e}}^{\text {hyd }}=6 \times 10^{-22} \mathrm{~m}^{2}$ et une diffusivité hydraulique de $\mathrm{k}^{\text {hyd }}=4 \times 10^{-7} \mathrm{~m}^{2} /$ jour.

On a alors considéré une caverne de forme sphérique, d'un volume de $33500 \mathrm{~m}^{3}$ à $1500 \mathrm{~m}$ de profondeur, lessivée en 100 jours, fermée puis abandonnée. On a simulé son comportement de très long terme en utilisant les deux jeux de paramètres qui avaient permis de décrire l'essai uniaxial. A la profondeur de la caverne la température était estimée à $60^{\circ} \mathrm{C}$, avec un gradient géothermique moyen de $0,03^{\circ} \mathrm{C} / \mathrm{m}$. La température du massif n'étant pas la même que celle de l'éprouvette. on a adopté une dépendance identique des paramètres A et $A^{*}$ avec la température:

$A(T)=A_{45^{\circ} \mathrm{C}} \exp \left\{-\frac{Q}{R}\left(\frac{1}{T}-\frac{1}{318}\right)\right\} \quad A^{\prime}(T)=A_{* 0 C}^{\prime} \exp \left\{-\frac{Q}{R}\left(\frac{1}{T}-\frac{1}{318}\right)\right\}$

où $Q / R=12600 \mathrm{~K}$ a la même valeur pour les deux modèles. De même il a fallu compléter les quatre constantes du modèle de M-D utilisées pour le modèle uniaxial par les deux nouvelles constantes $c=0,0092$ (qui décrit l'influence de la température sur la déformation $\varepsilon_{\mathrm{t}}^{*}$ ) et $\delta=0,58$ (qui décrit le comportement au déchargement). Ces deux constantes nouvelles n'ont toutefois pas un très grand poids dans l'évolution à long terme.

Les deux lois mécaniques de comportement donnent alors au comportement global de la caverne deux allures qualitativement distinctes :

- dans le cas de la loi de N-H (complétée par la loi de M-D pour la description de l'épisode transitoire) la pression dans la caverne croît lentement vers une valeur d'équilibre (un peu inférieure à $26 \mathrm{MPa}$ comprise entre la pression géostatique $(\mathrm{P} \infty=33 \mathrm{MPa})$ et la pression halmostatique $\left(\mathrm{P}_{\mathrm{h}}=18 \mathrm{MPa}\right)$. Cette pression

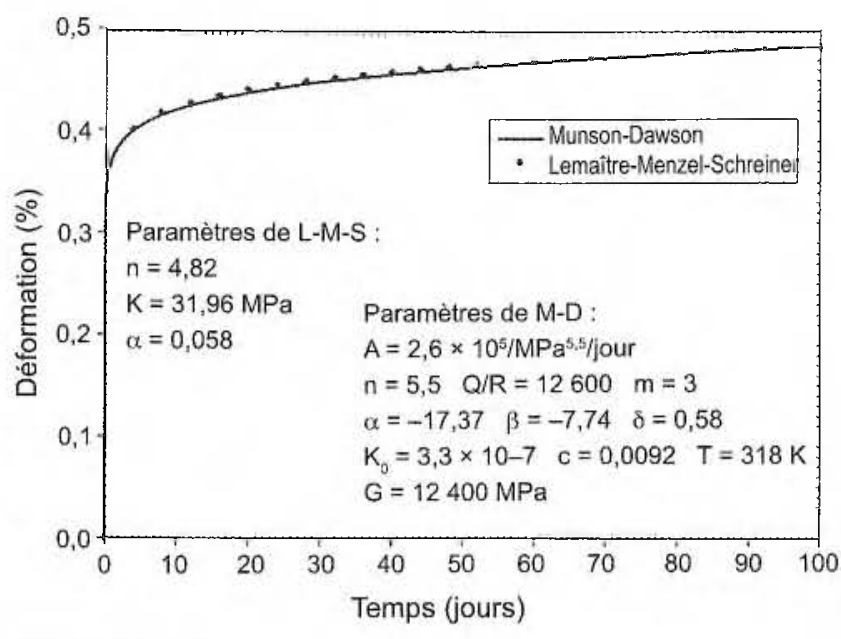

FIG. 7 Essai de fluage simulé par la loi de L-M-S et la loi de N-H et M-D. La charge sur l'éprouvette est $10 \mathrm{MPa}$ et la température est $45^{\circ} \mathrm{C}$.

A creep test simulated by the L-M-S law and the $\mathrm{N}-\mathrm{H}$ and $\mathrm{M}-\mathrm{D}$ law. The applied load is $10 \mathrm{MPa}$ and the temperature is $45^{\circ} \mathrm{C}$.

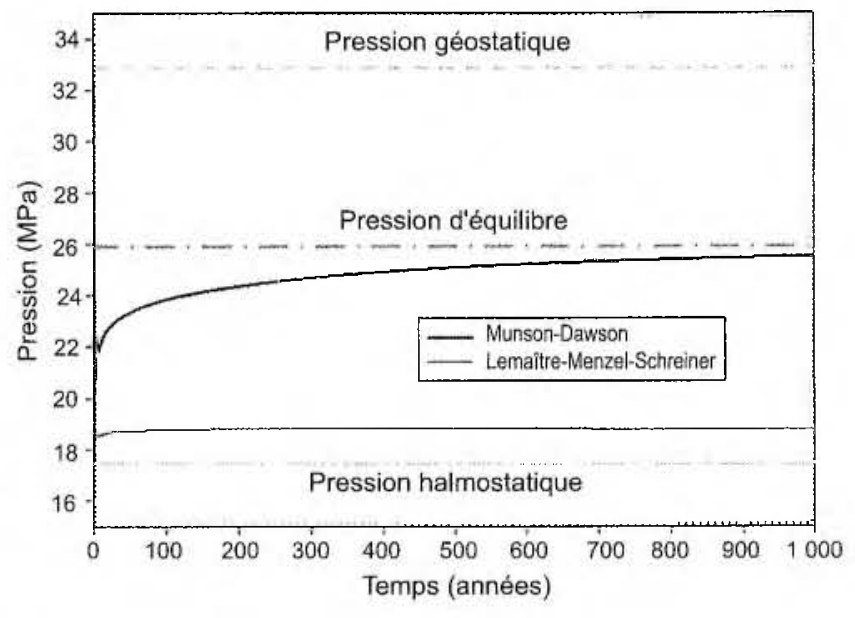

FIG. 8 Comportement à long terme de la pression dans une caverne fermée et abandonnée pour les deux modèles adoptés pour décrire Y'essai uniaxial représenté à la figure 7 .

Long-term behaviour of a sealed and abandoned cavern. The rheological models are identical to those considered when describing the uniaxial test shown on figure 7 .

d'équilibre, atteinte à mi-hauteur de la caverne, peut être considérée comme sûre car même au sommet de la caverne la pression de la saumure est significativement plus faible que les contraintes régnant dans les terrains à la même profondeur : le risque d'une fracturation naturelle peut être écarté. Ce critère serait plus difficilement vérifié par une cavité de grande hauteur et de grandes dimensions;

- dans le cas de la loi de L-M-S la pression dans la caverne reste beaucoup plus faible et décroît même après une période de 600 ans.

Les essais d'abandon réalisés dans des cavernes souterraines tendent à montrer l'existence d'une pression d'équilibre, comme le prévoit la loi de N-H (Bérest et al., 2001 ; Brouard consulting et al., 2006). Toutefois, ces essais ne constituent, pas plus que les autres essais en place décrits dans les paragraphes précédents, une 
preuve décisive : leur durée a été de un an et demi et de quatre ans, respectivement. A la fin de tels essais, les évolutions deviennent suffisamment lentes pour qu'une extrapolation à des durées de plusieurs siècles soit inévitablement incertaine. Le choix ne peut être dicté en définitive, dans l'état actuel des connaissances, que par une considération qui relève de la précaution : en l'absence d'arguments décisifs, il faut essayer les divers modèles et retenir le modèle qui donne les résultats les moins favorables. Pour le problème de l'abandon d'une caverne, c'est donc le modèle de N-H qui doit être retenu.

\section{0}

\section{Conclusion}

Il est difficile d'extrapoler à de très longues périodes de temps, de l'ordre du millénaire, les résultats d'essais de fluage conduits pendant une période de l'ordre d'une année. De tels essais peuvent être interprétés de manière également convaincante au moyen de lois de comportement différentes. En effet les essais de laboratoire et les mesures faites dans les ouvrages souterrains, étant affectés d'une incertitude résiduelle dont l'ordre de grandeur est comparable à l'écart entre les prévisions faites par les divers modèles, ne permettent pas de les discriminer. En revanche les modèles peuvent conduire à des conclusions très distinctes quant au comportement à long terme d'une cavité abandonnée.
Cette situation ne laisse pourtant pas complètement désarmé. On peut attendre un progrès régulier dans la qualité des mesures de laboratoire et en place, d'autant que ce problème de l'extrapolation est commun à de nombreux projets nouveaux d'utilisation du sous-sol (enfouissement de déchets, stockage du $\mathrm{CO}_{2}$ ) auxquels des moyens importants sont consacrés. La compréhension des micro-mécanismes à l'urigine des déformations des roches, servie par des moyens d'observation à diverses échelles, fait des progrès rapides.

Il faut aussi garder à l'esprit qu'une connaissance parfaite du comportement à long terme n'est pas toujours strictement indispensable. If faut vérifier, pour chaque ouvrage concret, les conséquences des incertitudes qui subsistent. On observe parfois que les objectifs de sécurité des personnes et de l'environnement peuvent rester satisfaits quand on conduit les calculs en faisant varier dans une très large gamme les valeurs des paramètres des modèles, ou même les modèles eux-mêmes. Les résultats des calculs permettent de pointer les incertitudes qui jouent un rôle majeur et de porter l'effort sur lá réduction de celles-ci.

Un tel processus exige la confrontation régulière des opinions, par les publications dans les revues et les réunions internationales. L'ambition du présent article est de contribuer à cette discussion.

Les auteurs remercient Gérard Vouille qui leur a fait part de nombreuses remarques et les colleguts des entreprises CSME, Gaz de Fiance, Geostock ef Total avec lesinuels ils ont partagé de fructueuses discussions et la conduite de plusjeurs essais en place.
} 
Abel I.-F. - "Rock Mechanics : can it pay its way? " Proceedings of the '3rd Symposium on Salt, Northern Ohio Geological society Inc., 1970, p. 197-207

Ait Chalal A. - « A comparison of the responses of the Munson-Dawson and the Lemaître Model ). Proceedings of the 3rd conference on the mechanical behavior of salt, Trans Tech Pub, 1996, p. 67-74.

Aubertin M., Sgaoula J., Servant S., Julien M.R. - " An up-to-date version of Suvic$\mathrm{D}$ for modelling the behaviour of salt $)$. Proceedings of the 4th conference on the mechanical behavior of salt, Trans Tech Puib, 1998, p. 205-220.

Baar C.A. - Applied salt-rock mechanics. Developments in geotechnical engineering. 16-A. Elsevier Science, vol. I, 1977.

Bérest P., Bergues J., Brouard B., Durup J.G., Guerber B. - « A salt-cavern abandonment test ». Int. J. Rock Mech. Min. Sci. no 38 (2), 2001, p. 343-355.

Bérest P., Brouard B., Feuga B. - "Abandon des mines de sel : faut-il ennoyer ? s. Revue française de géotechnique, n $106-$ 107,2004 , p. 53-71.

Bérest P., Blum P.A., Charpentier J.-P., Gharbi H., Valès F. - " Very slow creep tests on rock samples m. Int. J. Rock Mech. Min. Sci. $n^{\circ} 42,2005$, p. 569-576

Bérest P., Brouard B., Karimi-Jafari M., Van Sambeek L. - « Transient behaviour of salt caverns. Interpretation of Mechanical Integrity Tests m. Int. J. Rock Mech. Min. Sci., n ${ }^{\circ}$ 44, 2007, p. 767-786.

Bérest P - « Questions on the Prediction of the long-term behaviour of underground openings \%. Proceedings 11th ISRM Congress, Lisbonne, 2008, vol. 3, p. 14131425.

Blum W., Fleiscmann C. - « On the deformation-mechanism map of rock salt $)$. Proceedings of the 2nd conference on the mechanical behavior of salt, Trans Tech Publ., 1988, p. 7-22.

Brouard B., Bérest P. « A tentative classification of salts according to their creep properties ». Proceedings of the SMRI Spring Mecting, New Orleans, Louisiana, 1998, p. 18-38.

Brouard Consulting, Institute für Unterirdishches Bauen (IUB), École polytechnique, Total E\&P France, Géostock - Salt-Cavern Abandonment Field Test in Carresse. SMRI RFP 2003-2-B, Final Report, April 2006

Campos de Oreillana A.J. - « Pressure solution creep and non-associated plasticity in the mechanical behavior of potash mine openings u. Int. J. Rock Mech. Min. Sci., vol. $33, n^{\circ} 4,1996$, p. 347-370

Charpentier J.-P. « Creep of rock salt at elevated temperature m. Proceedings of the 2 nd conference on the mechanical behavior of salt, Trans Tech Pub., 1988, p. 131-136.

Clerc-Renaud A., Dubois D. - « Long-term operation of underground storage in salt ). Proceedings of the 5th Symposium on Salt, Northern Ohio Geological society Inc, vol. II, 1980 , p. 3-10

Cristescu N.D., Hunsche U. - Time Effects in Rock Mechanics. John Wiley \& Sons Itd, 1998.

DeVrjes K.L., Mellegard K.D., Callahan G.D. - "Cavern design using a salt damage criterion : proof-of-concept research final report ). Proceedings of the SMRI Spring Meeting, Houston, Texas, 2003, p. 1-18.
Durup J.G. - « Long term tests for tightness evaluations with brine and gas in salt ). SMRI Fall Meeting, Hannover, Germany, 1994

Durup G., Xu J. - « Comparative Study of Certain constitutive Laws used to describe the rheological deformation of salts ). Proceedings of the 3rd conference on the mechanical behavior of salt, Trans Tech Pub, 1996, p. 75-83.

Gaulke K, Rokhar R., Staudmeister K., Zander-Schiebenhofer D. - « Reassessment of the creep behaviour of the Rüstringen salt dome for optimization and future development of the crude oil cavern storage facility ». Proceedings of the SMRI Fall Meeting, Halifax, Canada, 2007, p. $155-172$

Horseman S.T. - « Moisture content. A major uncertainty in storage cavity closure prediction ). Proceedings of the 2 nd conference on the mechanical behavior of salt, Trans Tech Pub, 1988, p. 53-68.

Hugout B. - « Mechanical behavior of salt cavities -in situ tests- model for calculating the cavity volume evolution s. Proceedings of the 2nd conference on the mechanical behaviour of salt, Trans Tech Pub, 1988, p. 291-310.

Hunsche U., Schultze O. - « Effect of humjdity and confining pressure on creep of rock salt $)$. Proceedings of the 3 rd confercnce on the mechanical behaviour of salt Trans Tech Pub., 1996, p. 237-248.

Hunsche U., Schultze O. - « Humidity induced creep and its relation to the dilatancy ). Proceedings of the 5th conference on the mechanical behaviour of salt, Balkema, 2002, p. 73-87.

Karimi-Jafari M. - Sur le comportement transitoire des cavités salines protondes. Thèse de doctorat de l'École polytechnique, 12 novembre 2007

Laouafa F, Ghoreychi M. - « Salt pillar creep analysis m. Proceedings of Eurock 06, european regional ISRM symposium, Liège, Belgique, 2006, p. 381-388.

Langer M. - « The rheological Behaviour of Rock salt m. Proceedings of the 1st conference on the mechanical behavior of salt, Trans Tech Pub, 1984, p. 201-240

Lux K.H., Heusermann S. - « Creep tests on rock salt with changing load as a basis for the verification of theoretical material laws ». Proceedings of the 6th International Symposium on Salt. The Salt Institute, 1983, vol. I, p. 417-35.

Mandel J. - « Généralisation non linéaire des corps de Maxwell et de Kelvin. Fluage et relaxation ». Colloque international de rhéologie, Publications du CNRS, 1960.

Munson D.E., Dawson P.R. - « Salt consti tutive modeling using mechanism maps ). Proceedinos of the 1st Conference on the Mechanical Behavior of Salt. TransTech Pub, 1984, p. 717-737.

Munson D.E., Fossum A.F. - " Approach to first model prediction of measured WIPP in situ room closure in salt m. Proceedings of the 30th US symposium on Rock Mechanics, West Virginia University, 1989.

Nguyen Minh D., Pouya A. - « Une méthode d'étude des excavations souterraines en milieu viscoplasticue. Prise en compte d'un état stationnaire des contraintes $n$. Revue Française de Géotechnique, n 59 1992, p. 5-14.
Pennock G.M., Drury M.R., Peach C.J. et Spiers C.J. « The influence of water on deformation microstructures and textures in synthetic $\mathrm{NaCl}$ m. Journal of Structural Geology, n² 28, 2006, p. 1-14.

Perrier F., Morat P. Le Mouël J.-L. - « Pres sure induced temperature variations in an underground quarry $)$. Earth Planet Science Letters n 191, 2001, p. 145-156.

Popp T., Wiedeman M., Kansy A., Pusch G. - « Gas transport in dry rock salt-implications from laboratory investigations and field studies 1). Proceedings of the 6th conference on the mechanical behavior of salt, Taylor \& Francis Group, 2007, p. 17-26.

Pouya A. - «Correlation between mechanical behaviour and petrological properties of rock salt $\mathrm{n}$. Proceedings of the 32 nd US Symposium on Rock Mechanics, Balkema, 1991, p. 385-392.

Pouya A. - Comportement rhéologique du sel gemme. Application à l'étude des excavations souterraines. Thèse de doctorat de l'École nationale des ponts et chaussées, 18 octobre 1991.

Ratigan J.L., Van Sambeek K.L., De Vries K.L., Nieland J.D. - The infuence of seal design on the development of the disturbed rock zone in the WIPP alcove seal tests, RSI0400, Report prepared by RE/SPEC Inc. Rapid City, SD, for Sandia National Laboratories, Albuquerque, NM, 1991.

Ratigan J. - ( The SMRI cavern sealing \& abandonment research program Summary m. Proceedings of the SMRI Spring Meeting, Houston, Texas, 2003, p. 141-164.

Serata S. - " Application of continuum mechanisms to design of deep potash mines in Canada m. Int. J. Rock Mech. Min Sci., 1968, p. 293-314.

Spiers, C.J., Peach C.J., Brzesowsky R.H., Schutjens P.M.T.M, Liezenberg J. L., Zwart H.J. - Long Term Rheological and Transport Properties of Dry and Wet Salt Rocks, EUR 11848, prepared for Commission of the European Communities, by University of Utrecht, Utrecht, The Netherlands, 1988.

Tijani M. - «Zones de traction au cours d'es sais de compression m. Comptes rendus du $6^{c}$ Congrès de la Société internationale de mécanique des roches, Balkema, vol. 1, 1987 , p. $1279-1282$

Tijani S.M., Vouille G., Hugout B. - a Le sel gemme en tant que fluide visqueux $》$. Comptes rendus du $7^{\circ}$ Congrès de la Société internationale de mécanique des rochos, 1983, p. 241-246.

Van Sambeek L.L. - « Salt Pillar Design Equation ). Proceedings of the 4th conference on the mechanical behavior of salt. Trans Tech Pub, 1996, p. 495-508.

Van Sambeek L.L., Bérest P., Brouard B. Improvements in mechanical integrity tests for solution-mined caverns used for mineral production or liquid-product storage. SMRI Research Project Report 2005-1. 142 p

Vouille G., Tijani S.M, de Grenier F. - « Experimental determination of the rheological behavior of Tersanne rock salt ). Proceedings of the 1st conference on the mecha nical behavior of salt. Trans Tech Pub, 1984 p. $407-420$.

Wawersik W.R., Preece D.S. - « Creep testing of salt : Procedures, problems and sugges tions ). Proccedings of the 1st conforence on the mechanical bchavior of salt. Trans Tech Pub, 1984, p. 421-449. 